\title{
Training-induced neural plasticity in visual-word decoding and the role of syllables
}

\author{
Atsuko Takashima ${ }^{\mathrm{a}, \mathrm{d}, *}$, Barbara Wagensveld ${ }^{\mathrm{a}}$, Miranda van Turennout ${ }^{\mathrm{b}}$, \\ Pienie Zwitserlood ${ }^{\mathrm{c}}$, Peter Hagoort ${ }^{\mathrm{d}}$, Ludo Verhoeven $^{\mathrm{a}}$ \\ a Radboud University Nijmegen, Behavioural Science Institute, P.O. Box 9104, 6500 HE Nijmegen, The Netherlands \\ ${ }^{\mathrm{b}}$ Scientific Bureau of the Public Prosecution Service, Postbus 20305, 2500 EH The Hague, The Netherlands \\ ${ }^{\mathrm{C}}$ Institute for Psychology II, University of Münster, Fliednerstr. 21, 48149 Münster, Germany \\ ${ }^{\mathrm{d}}$ Radboud University Nijmegen, Donders Institute for Brain, Cognition and Behaviour, P.O. Box 9101, Kapittelweg 29, 6500 HB Nijmegen, The Netherlands
}

\section{A R T I C L E I N F O}

\section{Article history:}

Received 18 September 2013

Received in revised form

30 April 2014

Accepted 12 June 2014

Available online 21 June 2014

Keywords:

fMRI

Mental syllabary

Reading

Word learning

Pseudoword decoding

\begin{abstract}
A B S T R A C T
To investigate the neural underpinnings of word decoding, and how it changes as a function of repeated exposure, we trained Dutch participants repeatedly over the course of a month of training to articulate a set of novel disyllabic input strings written in Greek script to avoid the use of familiar orthographic representations. The syllables in the input were phonotactically legal combinations but non-existent in the Dutch language, allowing us to assess their role in novel word decoding. Not only trained disyllabic pseudowords were tested but also pseudowords with recombined patterns of syllables to uncover the emergence of syllabic representations. We showed that with extensive training, articulation became faster and more accurate for the trained pseudowords. On the neural level, the initial stage of decoding was reflected by increased activity in visual attention areas of occipito-temporal and occipito-parietal cortices, and in motor coordination areas of the precentral gyrus and the inferior frontal gyrus. After one month of training, memory representations for holistic information (whole word unit) were established in areas encompassing the angular gyrus, the precuneus and the middle temporal gyrus. Syllabic representations also emerged through repeated training of disyllabic pseudowords, such that reading recombined syllables of the trained pseudowords showed similar brain activation to trained pseudowords and were articulated faster than novel combinations of letter strings used in the trained pseudowords.
\end{abstract}

(c) 2014 Elsevier Ltd. All rights reserved.

\section{Introduction}

Can you pronounce "peemfesk"? Of course you can, even if you never saw this stimulus before. One way to do this is by converting each smallest unit - a grapheme, in our writing system - to a phoneme, as children often do when learning to read. The process of visual-word decoding, or the accurate and fast retrieval of the phonological code for written word forms, is commonly assumed to play a central role in the process of visual word recognition (Seidenberg, 2007). Even in adults, the initial stages of decoding novel words would involve the mapping of small units - be it individual graphemes to phonemes or bi- and trigrams to a (set of) phoneme(s). With repeated exposure, people become much faster at decoding words than it would take to decipher each grapheme separately (Nazir, Jacobs, \& O'Regan, 1998). A plausible mechanism

\footnotetext{
* Corresponding author at: Radboud University Nijmegen, Donders Institute for Brain, Cognition and Behaviour, P.O. Box 9101, Kapittelweg 29, 6500 HB Nijmegen, The Netherlands. Tel.: +312436 68064; fax: +31243610989.

E-mail address: atsuko.takashima@fcdonders.ru.nl (A. Takashima).
}

for this acceleration is that the units for the orthographyphonology mapping become increasingly larger, and the mapping becomes more holistic. Nonetheless, it is by no means clear how this orthography-phonology mapping is coded in our brain and whether the memory representation changes as a function of repeated exposure to a larger chunk of units (multisyllabic words).

To shed more light on the neurocognitive foundations of visualword decoding, we examined the underlying neuronal signature of reading aloud novel, disyllabic input strings, and how it changes as a function of repeated training over a period of four weeks. Each novel input string consisted of two syllables that do not exist in the language of our Dutch participants, but that could exist, because their combination of phonemes complies with the syllable-structure rules of their language. These strings had to be read aloud. Our aim was to study changes in the orthography-phonology mapping, and the emergence of units larger than individual graphemes or phonemes, as a function of training. Although the (neural) nature of visual word forms has been investigated (Dehaene, Cohen, Sigman, \& Vinckier, 2005; McCandliss, Cohen, \& Dehaene, 2003), not much is known yet about training-induced neural changes when decoding 
novel words. In a series of studies, Xue et al. trained Chinese participants to read unfamiliar Hangul characters, focusing on visual processing (Xue, Chen, Jin, \& Dong, 2006a, 2006b; Xue \& Poldrack, 2007) and how this training impacts on the involvement of the visual word form area (VWFA) in the left mid-fusiform gyrus (McCandliss et al., 2003). All studies by Xue et al. focused on visual processing and did not explore brain activation related to orthography-phonology conversion and motor preparatory processing. Several reading models suggest multiple processing steps that underlie reading, from decoding visual input, and transforming it to production output (for instance: Coltheart, 2005; Seidenberg, 2007). The models are usually built on how we decode combinations of different letter strings in a bottom-up manner. But they also imply that when a word holds semantic information, top-down modulation can affect the processing cascade, adding extra complexity to the models. Since we do not know much about training-induced neural plasticity in decoding unfamiliar script, here as a first step, we kept our focus simple by using pseudowords to avoid the top-down influence of the semantic representations.

In this study, we assessed the underlying neural mechanisms contributing to the decoding of visual input for articulation. Our assumption was that processing is demanding during the initial stage of decoding, when letter combinations are unfamiliar. This processing demand may arise because decoding unfamiliar letter combinations involves: 1) conversion of each grapheme to a phoneme, 2) keeping the converted information online in working memory, and 3) assembling the converted pieces to pronounce the letter strings as a whole. We assumed that repeated exposure to specific combinations of letter strings results in the emergence of more holistic representations at orthographic, phonemic, and motor-sequence levels. Once such holistic (syllable- or word-sized) representations exist, the second and the third decoding steps just mentioned can be dispensed with, since conversion will take place at a holistic level, rendering the decoding process more efficient.

Besides the learning processes involved in the decoding of visual pseudoword representations, we were also interested in whether repeated exposure to disyllabic pseudowords led to the formation of novel memory representations that were larger than individual phonemes, but smaller than the complete input string (i.e. the whole pseudoword). Following the idea put forward by statistical learning models (Barlow, 1989; Hastie, Tibshirani, \& Friedman, 2009), if a certain combination of letters occurs repeatedly, there is a high probability that this combination will occur again. The brain can code this set of letters as a chunk or unit, for efficient future usage. Likewise, phonological and motor representations can emerge as chunks through repeated training. By processing input information in chunks rather than individual graphemes, we assumed the decoding to be less effortful and more efficient. Since letter combinations occur more frequently as syllable packages when trained on a set of disyllabic pseudowords, we hypothesized that recombinations of these syllables will be decoded more efficiently than novel combinations at the letter level. It has been shown that syllables play a role in reading (Ans, Carbonnel, \& Valdois, 1998; Carreiras, Mechelli, Estévez, \& Price, 2007; Carreiras, Mechelli, \& Price, 2006; Carreiras, Riba, Vergara, Heldmann, \& Münte, 2009), and in speech production (Levelt, 2001; Schiller, Meyer, Baayen, \& Levelt, 1996). Levelt et al. put forward the idea of the "mental syllabary", a repository of articulatory phonetic programs for the most frequent syllables (Levelt, Roelofs, \& Meyer, 1999; Levelt \& Wheeldon, 1994), which facilitates the efficient production of speech. Applying these ideas to orthography-phonology mapping, we investigated whether specific memory representations for syllables will emerge as a consequence of repeated exposure to, and pronunciation of, multisyllabic words. If so, this would provide additional flexibility and efficiency in decoding. Therefore, we focused on the emergence of novel syllabic memory representations that, when extracted from the frequently exposed multisyllabic words, could be used in the processing of new combinations of these syllables.

In order to investigate the above objectives, we created a set of Dutch pronounceable disyllabic pseudowords which were comprised of two novel syllables (i.e. syllabic structures that conform to the rules of Dutch phonology, but do not exist in Dutch). The pseudowords were presented in Greek orthography to participants who all had basic training in reading ancient Greek texts, and were able to read aloud a short Dutch poem written in Greek script without any difficulty that was presented at the intake session prior to the experiment. Presenting the novel syllable combinations in the native Latin script invites access to native-language units that are larger than graphemes but smaller than syllables, whereas a grapheme-to-phoneme read out is possible in both Latin and Greek scripts. By using the Greek script, we hoped that participants are able to decode the letter strings if given enough time, but at the same time, to minimize the use of sub-syllabic mapping units that already exist in the native Latin script for decoding new Dutch-like pseudowords. Note that the novel syllables also did not exist in ancient Greek, and that additional non-Greek symbols had to be used to represent Dutch phonemes that did not exist in ancient Greek. The use of Dutch pronounceable pseudowords in a non-native script should thus render the decoding of the novel stimuli more difficult, and less automatic, and provide more opportunity to monitor the consequences of training, both in terms of general performance gain and the establishment of novel syllabic representations with behavioral measures and brain correlates.

The critical conditions consisted of "Trained" (five extensively trained combinations of 10 novel syllables; e.g. $\nu \omega \chi \beta \lambda_{1} \varphi, \pi \varepsilon \varepsilon \mu \varphi \varepsilon \sigma \kappa$, pronounced noogblif and peemfesk, respectively), and two additional conditions in which each stimulus was presented only once in every test session: "Recombined" (different combinations of the 10 syllables used in the trained condition; e.g. $\nu \omega \chi \varphi \varepsilon \sigma \kappa$, pronounced noogfesk), and "Novel" (novel pronounceable letter strings, the letters of which were used in the Trained pseudowords; e.g. $\nu \varepsilon \mu \sigma \lambda \varepsilon \varphi$, pronounced nemslef). We measured the brain activity using functional magnetic resonance imaging (fMRI) while participants were instructed to overtly pronounce the script presented on the screen. The stimuli were constructed such that grapheme-phoneme associations had a very transparent, one-toone correspondence (see Appendix Table A.1 for the graphemephoneme correspondence).

We predicted the following: during early stages of training, pseudowords will be decoded in small units in an effortful serial process, probably one grapheme at a time, causing errors at the grapheme/phoneme level, and relatively long response times. With repeated training, we expected the emergence of pseudoword representations, leading to substantial improvement in accuracy and speed for the Trained condition. On the neural level, we predicted an activation increase in areas that code for memory representations for the novel pseudowords as a whole, and for their constituent novel syllables. Because the naming task involved the conversion of visual input into phonological and motor output, we expected memory representations to arise in areas that code for visuo-auditory convergence, such as the posterior middle temporal gyrus (Hickok \& Poeppel, 2007), left temporo-parietal regions, and superior temporal cortex (Wilson, Isenberg, \& Hickok, 2009). We also predicted activation decrease with training, in areas such as the occipito-parietal cortex, known to be active when the task demands extra attention to the visual input (Cohen, Dehaene, Vinckier, Jobert, \& Montavont, 2008; Kravitz, Saleem, Baker, \& Mishkin, 2011; Sandak et al., 2004), and in the prefrontal cortex, whose activity reflects conscious selection and monitoring (Graves, Desai, Humphries, Seidenberg, \& Binder, 2010). The same holds for areas known to activate when articulation is 
difficult, such as the anterior insula and the premotor cortex (Brown et al., 2009). If syllable-sized representations are indeed established, Recombined pseudowords should be articulated faster than Novel pseudowords, and the brain activity in the Recombined condition should be similar to that of Trained condition, as these two conditions share the same syllables.

\section{Materials and methods}

\subsection{Participants}

Twenty-one right-handed university students from the Radboud University Nijmegen (four males, age $18-29$ years, $M=21.2, \mathrm{SD}=2.6$ ) participated in the study. All were native speakers of Dutch, studying Classical Languages at the university All participants were without any known neurological or hearing problems and with normal or corrected to normal vision. Participants gave written informed consent according to the Declaration of Helsinki and were compensated for participation. To be confident that the participants could perform the task already on Day 1, at the intake of the experiment they were given a short Dutch poem written in Greek script to read aloud, and all could do this without difficulty.

\subsection{Stimulus}

Stimuli consisted of disyllabic Dutch pseudowords, comprised of novel Dutch syllables (i.e. pronounceable strings of phonemes that conform to the rules of Dutch phonology, but do not exist as a syllable, or a part of a syllable, in Dutch) and assigned to one of three experimental conditions. For the Trained condition, 10 novel syllables were used to form five pseudowords (e.g. noogblif and peemfesk). For the Recombined condition, the 10 novel syllables of the Trained condition were paired anew, to form 70 recombined pseudowords (e.g. noogfesk and blifpeem). For the Novel condition, 140 new disyllabic pseudowords were created using the same graphemes as were used in the Trained condition (e.g. nemsslef and feepslif). Of the 140 pseudowords in the Novel condition, 50 were assigned to the Novel condition in the functional magnetic resonance imaging (fMRI) test and 20 to the Novel condition in the Behavioral test on Day 1. Of the remaining 70 pseudowords in the Novel condition and of the 70 in the Recombined condition, 50 were assigned to the fMRI test list and 20 to the Behavioral test list for both Days 5 and 28. The visual baseline condition during the fMRI test consisted of "XXXXXXX" on the screen. The length of the sequences was matched to the length of the experimental stimuli. Pseudowords were presented in Greek script (e.g. $\nu \omega \gamma \beta \lambda_{1 \varphi}$ for noogblif and $\pi \varepsilon \varepsilon \mu \varphi \varepsilon \sigma \kappa$ for peemfesk) in white on a black background. Three non-Greek symbols were used for the Latin graphemes that did not have Greek counterparts. Participants learned these exceptions by heart prior to the start of the experiment. Grapheme-phoneme correspondence was very transparent such that there was only one possible way to pronounce the script of each pseudoword. Greek and Non-Greek symbols and their corresponding Latin graphemes, as well as all stimuli, can be found in the Appendix Tables A.1 and A.2, respectively).

\subsection{Procedure}

Word-decoding and speech-production learning were established by training participants to overtly pronounce pseudowords, presented in a Greek script on a screen, through repeated sessions spread over four weeks (Fig. 1A). One day prior to the start of the experimental sessions, participants were given a table of symbolsound conversion (Appendix Table A.1) and some example texts to practice converting Greek to Latin format, including the three unfamiliar non-Greek symbols. The experiment encompassed three types of sessions: Training, fMRI test, and Behavioral test sessions. The experimental protocol was as follows: on Day 1, participants first took fMRI test 1 (fMRI-1) followed by Behavioral test 1(Behav-1), and then Training session 1 (Train-1). On Day 3, the second Training session took place (Train-2). On Day 5, participants took the second fMRI (fMRI-2) and Behavioral test (Behav-2). Eight Training sessions followed during the next three weeks (Train-3 to 10). Each Training session was maximally three days apart. On Day 28 (the last day of the experimental protocol), participants took the third fMRI (fMRI-3) and Behavioral test (Behav-3) (Fig. 1A).

During the fMRI test on Day 1, participants were presented with 100 pseudowords (presentation time varied from 2.5 to $6 \mathrm{~s}$ ) while they were scanned in an MR scanner ( $3 \mathrm{~T}$, Trio, Siemens, Erlangen) and were told to pronounce the pseudowords. These 100 trials consisted of 10 repetitions of the 5 pseudowords from the Trained list and 50 different pseudowords from the Novel list. Participants were instructed to pronounce the visually presented stimuli overtly, as soon as an asterisk (*) appeared on the screen. For every trial, the pseudoword appeared in white letters on a black background. After a jittered interval of 2.5-6 s, it was replaced by an asterisk, prompting the participants to pronounce the pseudoword, followed by a jittered inter-trial interval of 2-10 s. Intermixed, 50 visual baseline stimuli ("XXXXX") were presented on the screen, and participants were instructed to just passively view the stimulus (Fig. 1B: fMRI). Both jittered intervals were used to prevent stimulus and response-time anticipation. All stimuli were presented in a random order. On Day 5 and Day 28, the stimulus set of the fMRI test comprised: 10 repetitions of the 5 pseudowords from the Trained list, 50 new pseudowords from the Novel list, and a set of the 50 pseudowords from the Recombined list, adding to a total of 150 pseudoword trials and 50 baseline trials per session.

After each fMRI test session, participants performed a Behavioral test session (Fig. 1B: Behav). During this session, 4 repetitions of the 5 pseudowords of the Trained condition, 20 Novel pseudowords, and on Days 5 and 28, an additional 20 pseudowords from the Recombined condition were presented visually to the participants in a randomized order. There were thus 40 trials on Day 1, and 60 trials on Day 5 and Day 28. Participants were instructed to overtly pronounce the pseudowords as quickly and as accurately as possible.

In all 10 training sessions, participants were visually presented with the 5 pseudowords from the Trained list 200 times each, in a randomized order, and they were instructed to overtly pronounce the stimuli as quickly and as accurately as possible (Fig. 1B: Train).

\subsection{Behavioral data analysis}

Accuracy and reaction times were measured during the Behavioral test sessions and compared using PASW Statistics 18, Release Version 18.0.0 (D3 SPSS, Inc., 2009, Chicago, IL, www.spss.com). Responses were marked as errors when they contained pronunciation errors, or were not initiated within the time window of 200$1750 \mathrm{~ms}$ after stimulus onset. Reaction time was defined as the time between the onset of the pseudoword presentation on the screen and the onset of the vocal naming response, measured by a voice key, adjusted to participants' individual voice amplitude levels, and connected with the experimental setup (NESU, MPI for Psycholinguistics, Nijmegen, The Netherlands http://www.mpi.nl/world/tg/experi ments/nesu.html for the first nine participants, and Presentation, Neurobehavioral systems https://www.neurobs.com/ for the remaining participants). Response times that were shorter than 200 ms (mean number of trials on Day 1: 0.2, Day 5: 0.4, Day 28: 0.9) and longer than the response-time limit (1750 ms, mean number of trials on Day 1: 1.9, Day 5: 0.6, Day 28: 0.6) were omitted from the reaction-time analysis. For repeated measures analyses of variance (ANOVA), Greenhouse-Geisser correction was applied whenever sphericity was violated.

\section{5. fMRI data acquisition}

For the fMRI, we acquired T2*-weighted images covering the whole brain using an echo-planar imaging sequence (EPI, 36 axial slices, ascending slice acquisition, repetition time $(T R)=2310 \mathrm{~ms}$, echo time $(T E)=30 \mathrm{~ms}, 75^{\circ}$ flip-angle, matrix $=64 \times 64$, slice thickness: $3.0 \mathrm{~mm}$, slice gap: $0.3 \mathrm{~mm}$, field of view (FOV): $192 \mathrm{~mm}$, Trio, Siemens, Erlangen). In order to minimize the effect of head motion due to word production, we made use of the online motion correction algorithm (Prospective Acquisition CorrEction, PACE) embedded in the Siemens' sequence, where the actual acquisition slice in the scanner is shifted according to online prospective motion correction of EPI images. For the structural MRI, we acquired T1-weighted images using a magnetization-prepared, rapid acquisition gradient echo sequence (MP-RAGE, 192 sagittal slices, TR $=2300 \mathrm{~ms}$, TE $=3.93 \mathrm{~ms}, 15^{\circ}$ flipangle, matrix $=256 \times 256$, slice thickness: $1.0 \mathrm{~mm}$, FOV: $256 \mathrm{~mm}$ ).

\subsection{MRI data analysis}

Image pre-processing and statistical analysis was performed using SPM8 (www.fil. ion.ucl.ac.uk). The first three volumes of each participant's functional EPI-data were discarded to allow for T1 equilibration. The EPI images were realigned to the first volume, and the subject mean was co-registered with the corresponding structural MRI using mutual information optimization. Both functional and structural scans were spatially normalized and transformed into a common Montreal Neurological Institute space (resampled at voxel size $2 \times 2 \times 2 \mathrm{~mm}^{3}$ ), as defined by the SPM8 T1.nii template, as well as spatially filtered by convolving the functional images with an isotropic 3D Gaussian kernel ( $8 \mathrm{~mm}$ full width at half-maximum, FWHM).

The fMRI data were analyzed statistically using the general linear model (GLM) and statistical parametric mapping. Five (Day 1) or seven (Day 5 and Day 28) explanatory variables were included in the model: Trained (word presentation, word articulation), Novel (word presentation, word articulation), and baseline for Day 1, and additional two regressors for Recombined (word presentation, word articulation) for Day 5 and Day 28. For word presentation and baseline regressors, the event was time-locked to the presentation of the visual stimulus, and for the word-articulation regressors, the event was time-locked to the offset of the pseudoword (when an asterisk appeared, prompting the subject to articulate). These explanatory variables were temporally convolved with the canonical hemodynamic response function, along with their temporal derivatives (one for each explanatory variable) provided by SPM8. The design matrix also included six head motion regressors ( 3 translations, 3 rotations) as covariates of no interest. A high-pass filter was implemented using a cut-off period of $128 \mathrm{~s}$ to remove lowfrequency effects from the time series. For statistical analysis, relevant contrast parameter images were generated for each participant and subsequently subjected to 
A

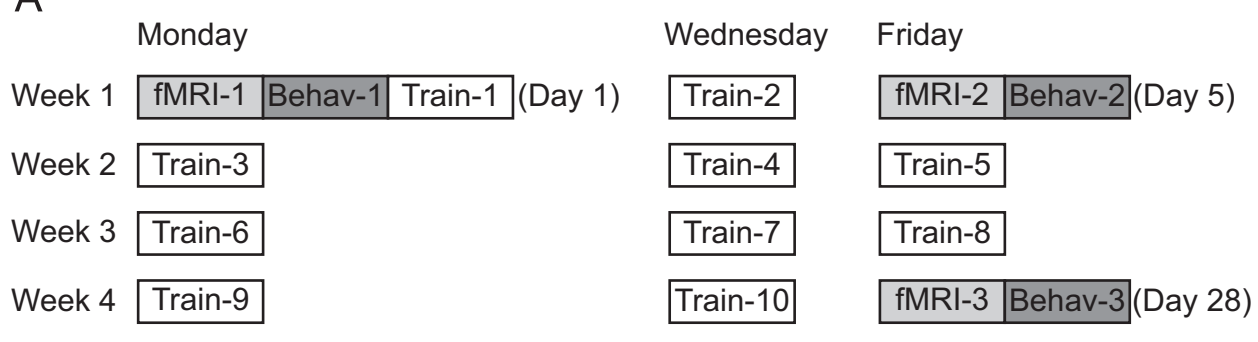

$\mathrm{B}$

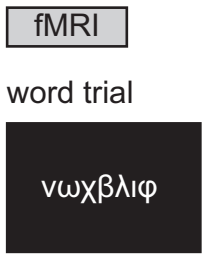

$2.5-6 s$

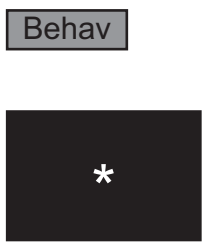

$250 \mathrm{~ms}$

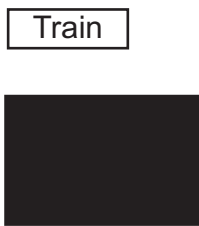

$850 \mathrm{~ms}$

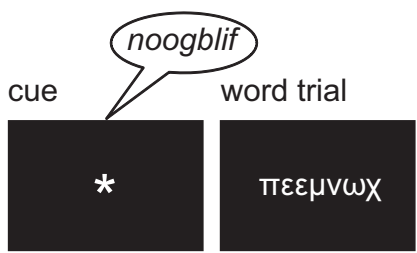

$2-10 s$

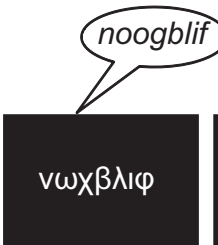

$700 \mathrm{~ms}$

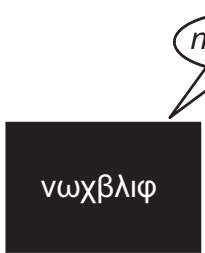

$500 \mathrm{~ms}$
$2.5-6 s$

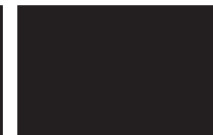

$1100 \mathrm{~ms}$

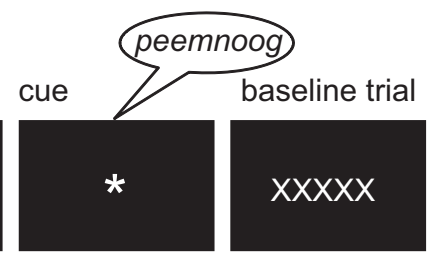

$2-10 s$

$2.5-6 s$

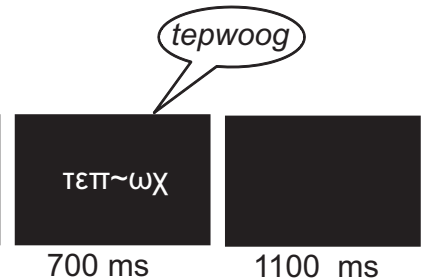

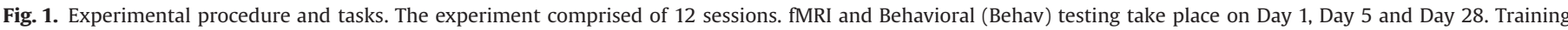

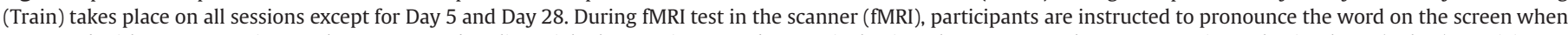

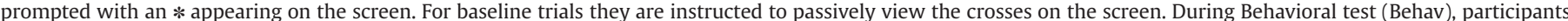

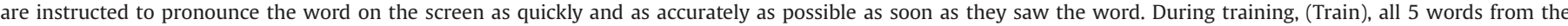
Trained condition are presented 200 times each in a random order. Participants are instructed to pronounce the word as quickly and as accurately as possible.

a second-level analysis (Penny, Holmes, \& Friston, 2003), treating participants as a random variable.

For our current purposes, we focused on activation related to the preparation of word pronunciation. For this reason, comparisons on the regressors pertaining to word presentation for the three conditions of interest were investigated. For withinsession comparisons, specific contrasts between conditions (word presentation for Trained-Novel, Trained-Recombined, and Recombined-Novel) for every participant were created within each session, and these contrast images were tested against 0 in one-sample $t$-test on the group level. For comparison between sessions of a specific condition, each condition was contrasted against the session baseline per participant, and these contrasts were compared using paired $t$-tests on the second level. Since the Trained pseudowords were novel in the beginning but became familiar with repetition during the fMRI test on Day 1, an additional model was tested for Day 1, including a parametric modulation regressor, accounting for the number of repeats in the Trained condition. All reported clusters are significant at voxel level $p<.05$ family-wise error corrected (FWE) based on random field theory (Brett, Penny, \& Kiebel, 2004) unless otherwise stated.

\section{Results}

The main questions addressed by the behavioral and fMRI data concerned: (1) effects of training, both short- and long-term, on accuracy and speed of naming, and on the brain networks involved in decoding and preparing to articulate stimuli; (2) the behavioral and neural consequences of recombining parts of the trained stimuli. The
Recombined condition involved new combinations of the syllablesized parts of the trained stimuli, which were never seen and spoken in this specific combination before the test on Day 5 .

\subsection{Behavioral results}

During fMRI test in the scanner, pseudoword production was a delayed response (participants had to withhold their naming response until they were cued by an asterisk appearing on the screen). Moreover, naming responses were contaminated by scanner noise. For these reasons, we focus on the data from the Behavioral test to assess changes in accuracy and speed as a function of time and training.

\subsubsection{Accuracy}

Accuracy for the Trained stimuli was already high on Day 1, and remained high throughout the experiment (Fig. 2A). The Recombined condition also showed a very few errors, but for the Novel condition, error rate was high in all three sessions. This was confirmed in two repeated measures ANOVAs. The first, with 3 sessions (Day 1, Day 5, Day 28) and 2 conditions (Trained, Novel) as factors revealed main effects of sessions $\left(F_{(2,40)}=10.27, p<.001\right)$, and of conditions 

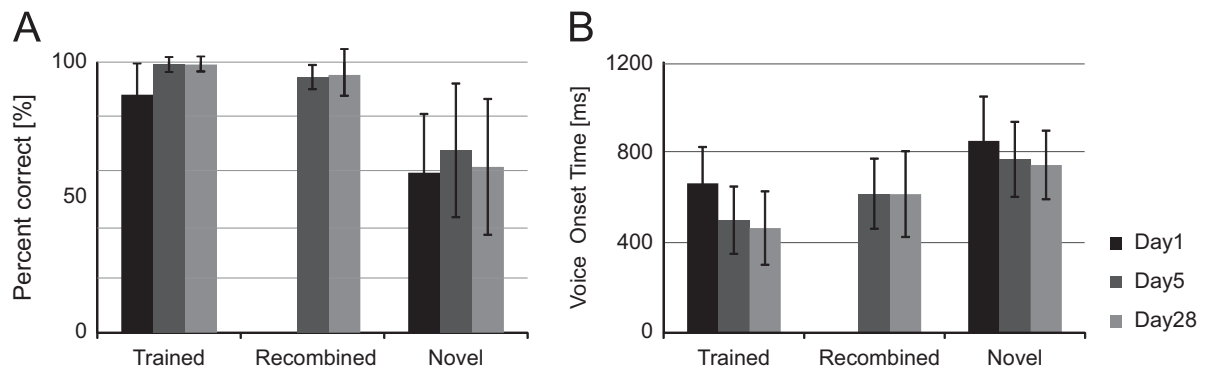

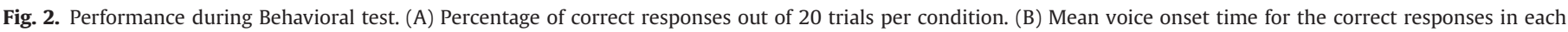
condition. Error bars represent the standard deviations.

$\left(F_{(1,20)}=51.20, p<.001\right)$. Post-hoc paired $t$-tests (Bonferroni corrected) showed a significant difference between Day 1 and Day $5(p<.001)$, and a trend between Day 5 and Day $28(p=.069)$. More errors were observed in the Novel condition in all sessions (all $p<.001$ ). The second ANOVA, with 2 sessions (Day 5 and Day 28) and all 3 conditions (Trained, Recombined, Novel) showed a main effect of conditions $\left(F_{(2,40)}=44.12, p<.001\right)$, and a marginal significance of the sessions $\times$ conditions interaction $\left(F_{(2,40)}=3.33, p=.063\right)$. Post-hoc paired $t$-tests (Bonferroni corrected) showed that for both Day 5 and Day 28, participants made more errors in the Novel condition than the Trained and Recombined conditions (all $p<.001$ ). Accuracy of the Recombined condition was significantly lower than the Trained condition on Day 5 $(p<.001)$ and showed a trend on Day $28(p=.099)$.

\subsubsection{Reaction time (voice-onset time)}

The Trained condition was always responded to fastest, followed by the Recombined, and then the Novel condition. Reaction times decreased across sessions for the Trained stimuli, but remained stable from Day 5 to Day 28 for the Recombined and the Novel stimuli (Fig. 2B). This was corroborated in similar ANOVAs as for the accuracy data.

The first ANOVA ( 3 sessions $\times 2$ conditions: Trained and Novel) revealed a main effect of sessions $\left(F_{(2,40)}=26.48, p<.001\right)$ and conditions $\left(F_{(1,20)}=178.18, p<.001\right)$ and an interaction $\left(F_{(2,40)}=8.19\right.$, $p=.002$ ). Pairwise contrasts (Bonferroni corrected) showed that Day 1 was slower than both Day 5 and Day 28 (both $p<.001$ ), and a trend towards Day 5 being slower than Day 28 ( $p=.094)$. The main effect of conditions was also significant, with faster responses in the Trained than in the Novel condition $\left(F_{(1,20)}=178.18, p<.001\right)$. Finally, the significant interaction corroborated that the decrease in the reaction time was driven by decrease in reaction time for the Trained condition (all $p<.05$ ), whereas the decrease for Novel condition only showed a trend between Day 1 and Day $28(p=.099)$. The second ANOVA, comparing all three conditions on Day 5 and Day 28, revealed a main effect of conditions $\left(F_{(2,40)}=142.56, p<.001\right)$. There was a linear decrease in reaction time, with the Trained condition being fastest and the Novel condition being slowest. No main effect of sessions or interaction was observed.

In summary, participants were fastest and most accurate at naming the Trained items. The Recombined stimuli were named slightly less accurately than Trained items, but performance in both the Trained and Recombined conditions was clearly better and faster than on the Novel items. Through training, participants became faster in naming the items in the Trained condition over the three test sessions; however, this effect could not be observed for the Recombined and Novel conditions from Day 5 to Day 28.

\subsection{Imaging results}

Brain activity data were compared within and across sessions for different conditions. The data are reported according to the questions raised above: (1) long- and short-term effects of training and (2) the neural consequences of recombining syllables of the trained disyllabic pseudowords into novel syllable combinations.

\subsubsection{Activity change over time with extensive training}

To investigate brain-network changes as a function of extensive training, we looked into the brain-activity difference between Day 1 and Day 28. Paired $t$-test comparison of contrasts for the Trained vs baseline between Day 1 and Day 28 showed an increase in activity as a result of training (Day $1<$ Day 28 ) in the bilateral angular gyrus (AG) and the left precuneus (Table 1 and Fig. 3A). No significant increase above the threshold was observed from Day 1 to Day 5, although the bilateral AG showed this trend.

Changes over time in particular brain regions may reflect the fact that the participants become acquainted with performing the task in the scanner. To confirm that changes in the areas reported above were due to training on specific pseudowords rather than to task familiarity, general effects of the task were also tested. To do so, the Novel-baseline contrasts were compared for Day 1 and Day 28. We did not observe any voxel that survived the threshold $\left(P_{F W E}<.05\right.$ on the voxel level) for increase over time. We also applied a cluster-size statistics (Hayasaka \& Nichols, 2003), using initial voxel level threshold at $p<.001$ and cluster-size $P_{F W E}<.05$ to detect activation patterns that might not be robust enough to be captured at voxel-level FWE corrected threshold. This comparison revealed two clusters; a bilateral precuneus cluster (local maximum $[4,-54,40])$ extending to the middle cingulate cortex, and a right $A G$ cluster (local maximum $[58,-58,30]$ ) extending to the inferior parietal lobe (IPL) that increased in activity with time. Even though there was a general increase in activity with training for both the Trained and the Novel conditions in the AG and the precuneus, when the areas that increased in activity from Day 1 to Day 28 in the Novel condition were masked out (i.e. excluding all significant voxels from the contrast Novel condition Day $28>$ Day 1 ), significant voxels in the bilateral AG (right local maximum $[56,-50,30]$ and left $[-54,-62,26]$ ) and in the precuneus (local maximum $[-4,-58,42]$ ) were found for the Trained condition.

Furthermore, direct comparison between the Trained and the Novel condition on Day 28 showed more activity for the Trained than the Novel condition in the bilateral AG cluster extending to IPL, and extending to the supramarginal gyrus of the right hemisphere (Table 2 and Fig. 4 top row). Moreover, this direct comparison (Day 28 Trained > Novel) also showed activity in the bilateral middle temporal gyrus (MTG) and the left superior occipital gyrus. Thus, the activity increase in these areas can be seen as the result of changes due to training on specific pseudowords rather than to task practice.

Decreases in activity with repeated training over one month (i.e. Trained condition: Day $1>$ Day 28) were observed in the bilateral ventral visual pathway, extending from the occipital cortex to the inferior temporal and fusiform gyri, and bilaterally 
Table 1

Activity change Day 1 vs Day 28 for Trained condition

\begin{tabular}{|c|c|c|c|c|c|c|c|}
\hline & \multirow[b]{2}{*}{ Cluster size } & \multicolumn{3}{|c|}{ Peak voxel } & \multicolumn{3}{|c|}{ MNI coordinates } \\
\hline & & $P_{F W E}$ & $\mathrm{~T}$ & $\mathrm{Z}$ & $\mathrm{X}$ & Y & $\mathrm{Z}$ \\
\hline \multicolumn{8}{|c|}{$\begin{array}{l}\text { Increase in activity with training (Day } 1<\text { Day 28) } \\
\text { (Voxel-level FWE corrected) }\end{array}$} \\
\hline right angular gyrus & 57 & 0.001 & 9.45 & 5.77 & 54 & -52 & 30 \\
\hline left angular gyrus & 68 & 0.002 & 8.45 & 5.45 & -54 & -62 & 26 \\
\hline left precuneus & 9 & 0.03 & 6.86 & 4.86 & -4 & -58 & 42 \\
\hline \multicolumn{8}{|c|}{$\begin{array}{l}\text { Decrease in activity with training (Day } 1>\text { Day 28) } \\
\text { (Voxel-level FWE corrected) }\end{array}$} \\
\hline left inferior occipital gyrus & 75 & 0.003 & 8.35 & 5.42 & -42 & -78 & -10 \\
\hline left inferior temporal gyrus & & 0.042 & 6.66 & 4.78 & -48 & -64 & -10 \\
\hline left fusiform gyrus & 4 & 0.034 & 6.79 & 4.83 & -30 & -62 & -4 \\
\hline right inferior temporal gyrus & 135 & 0 & 9.84 & 5.88 & 48 & -64 & -10 \\
\hline right fusiform gyrus & 3 & 0.042 & 6.65 & 4.78 & 44 & -62 & -20 \\
\hline left superior parietal lobule & 260 & 0 & 10.17 & 5.97 & -24 & -56 & 58 \\
\hline left inferior parietal lobule & & 0.005 & 7.96 & 5.28 & -28 & -54 & 48 \\
\hline right angular gyrus & 182 & 0.001 & 9.27 & 5.71 & 28 & -66 & 46 \\
\hline right superior parietal lobule & 2 & 0.038 & 6.71 & 4.8 & 28 & -56 & 62 \\
\hline left superior frontal gyrus & 18 & 0.014 & 7.33 & 5.05 & -24 & -6 & 54 \\
\hline left inferior frontal gyrus (pars opercularis) & 85 & 0.016 & 7.23 & 5.01 & -46 & 4 & 28 \\
\hline \multicolumn{8}{|c|}{ Increase in activity with training (Day $1<$ Day 5 ) } \\
\hline \multicolumn{8}{|c|}{ no suprathreshold voxels at $\mathrm{P}_{\mathrm{FWE}}<0.05$} \\
\hline left inferior occipital gyrus & 36 & 0.011 & 7.49 & 5.11 & -42 & -74 & -8 \\
\hline left middle occipital gyrus & 35 & 0.002 & 8.63 & 5.51 & -36 & -86 & 16 \\
\hline left inferior temporal gyrus & 1 & 0.046 & 6.59 & 4.75 & -50 & -50 & -14 \\
\hline right inferior temporal gyrus & 234 & 0.002 & 8.64 & 5.52 & 48 & -64 & -8 \\
\hline left inferior parietal lobule & 839 & 0 & 10.56 & 6.07 & -42 & -38 & 50 \\
\hline left superior parietal lobule & & 0.003 & 8.26 & 5.39 & -32 & -60 & 48 \\
\hline left superior parietal lobule & 3 & 0.038 & 6.70 & 4.80 & -36 & -58 & 60 \\
\hline right superior occipital gyrus & 343 & 0.003 & 8.25 & 5.39 & 30 & -66 & 42 \\
\hline right inferior parietal lobule & & 0.005 & 7.97 & 5.29 & 28 & -56 & 54 \\
\hline right superior parietal lobule & & 0.006 & 7.80 & 5.23 & 30 & -54 & 62 \\
\hline left precentral gyrus & 397 & 0 & 9.67 & 5.83 & -44 & -8 & 34 \\
\hline left inferior frontal gyrus (pars opercularis) & & 0.005 & 7.91 & 5.27 & -58 & 8 & 20 \\
\hline left precentral gyrus & 39 & 0.009 & 7.58 & 5.15 & -30 & -8 & 50 \\
\hline left supplementary motor area & 32 & 0.018 & 7.17 & 4.99 & -8 & 4 & 54 \\
\hline right supplementary motor area & & 0.042 & 6.65 & 4.94 & 2 & 6 & 52 \\
\hline left putamen & 1 & 0.045 & 6.60 & 4.76 & -24 & 8 & 0 \\
\hline left middle frontal gyrus & 2 & 0.022 & 7.05 & 4.94 & -38 & 48 & 12 \\
\hline vermis & 113 & 0.001 & 9.01 & 5.63 & 2 & -62 & -38 \\
\hline vermis & & 0.029 & 6.87 & 4.87 & -2 & -70 & -32 \\
\hline
\end{tabular}

FWE: family-wise error, MNI: Montreal Neurological Institute

in the superior parietal lobe, with left lateralized extensions to the inferior parietal lobe and inferior and superior frontal gyri (IFG, SFG) (Table 1 and Fig. 3B). Smaller but similar differences in the same areas were observed for the contrast Day $1>$ Day 5 (Table 1 ). Activity difference measures between the Novel and Trained conditions on Day 28 (Novel > Trained) showed more activity in bilateral visual areas, extending ventrally to the inferior temporal gyrus and fusiform gyrus, and dorsally to the middle occipital gyrus and superior parietal lobe, and further to the left precentral, the supplementary motor area, and SFG. This suggests a role for these areas in unfamiliar pseudoword decoding and articulation preparation, even when the task has become very familiar (Fig. 4 top row; for the whole list of activation clusters, see Table 2).

\subsubsection{Activity change as a function of repetition on Day 1}

Exposure to each Trained pseudoword for 10 times, during the fMRI test on Day 1, resulted in a performance advantage in the Behavioral test for the Trained pseudowords compared to that of the Novel pseudowords. This suggests a rapid learning effect of the Trained pseudowords during the fMRI test on Day 1. To see the change over repetitions for the Trained condition during the fMRI scan, we ran a separate GLM analysis including repetitions as a parametric modulator for the Trained condition in the model for the data on Day 1, and investigated the beta-values of this parametric modulator regressor. This analysis revealed a network of regions that increased in activity, and another network that decreased in activity with repetition. Not surprisingly, the areas that increased in activity with repetition were found in the left AG extending to the IPL, and in the right hemisphere AG extending to the supramarginal gyrus and IPL, and the MTG; very similar to the network that showed an increase over a month of training. The areas that decreased in activity with repetition were found in the left IPL and pre-central gyrus. To detect activation patterns that might not be robust enough to be captured at a voxel-level FWE-corrected threshold, we also investigated the activation map using cluster-level statistics with initial voxel level threshold at $p<.001$, and corrected for multiple comparisons on the cluster-size at $P_{F W E}<.05$. Additional increases in activation with repetition were found in the midline structures, including the precuneus extending to the posterior and middle cingulate cortex and the right SFG. With this statistical threshold, additional 
A

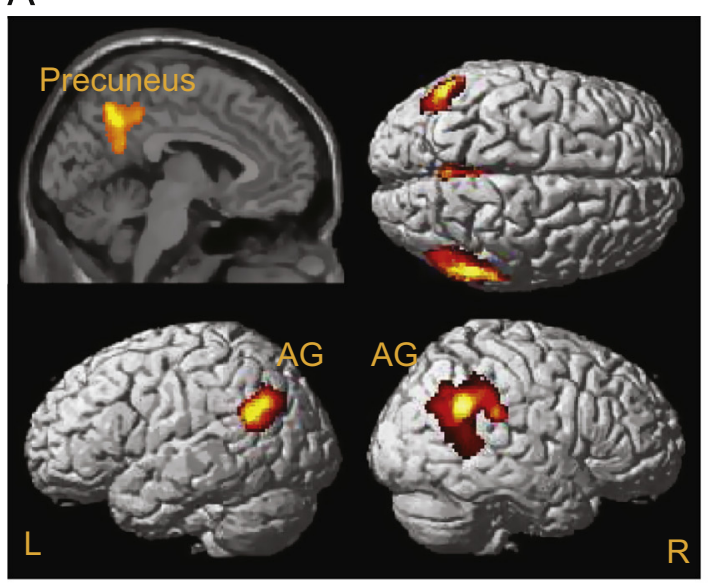

B

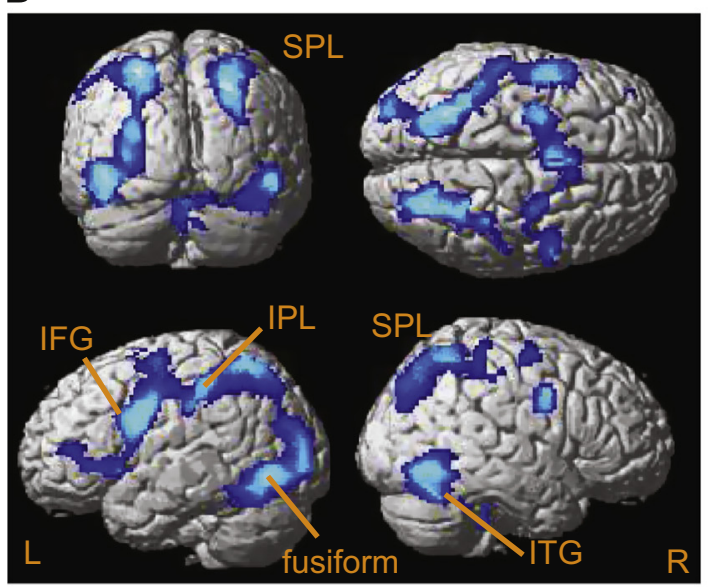

C
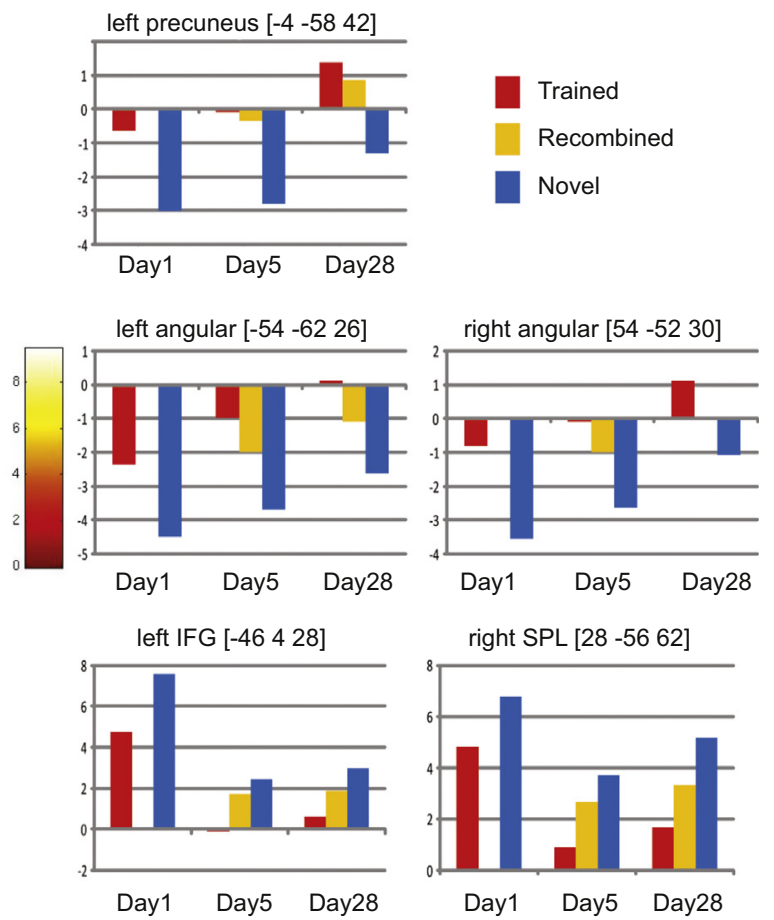

right SPL [28 -56 62]

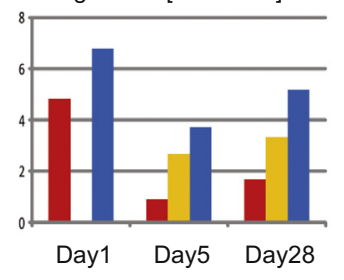

left fusiform [-30 -62 -4]

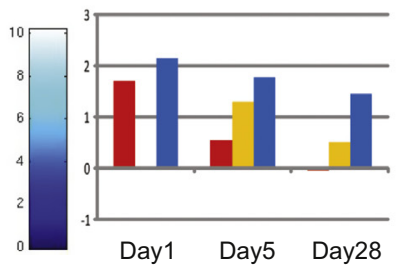

right ITG [48 -64 -10]

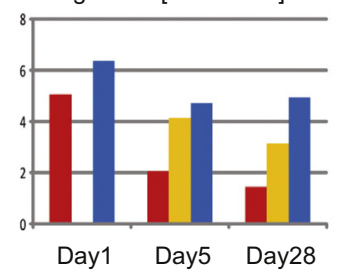

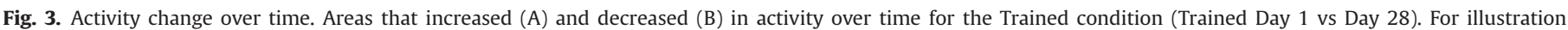

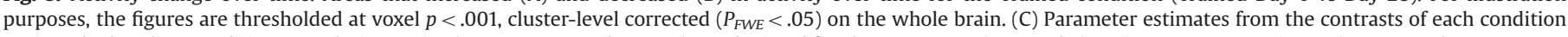



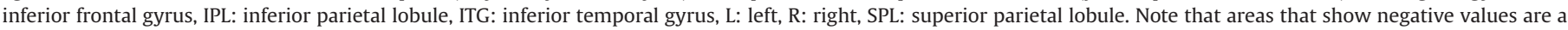

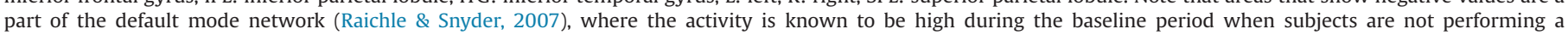
specific task.

decrease due to repetition was observed in the bilateral visual areas, extending ventrally to the right inferior temporal gyrus and dorsally to the postcentral gyrus, and in the left IFG (pars opercularis) (Fig. 5 and Table 3).

In summary, on Day 1, the networks observed for activation increase and decrease with repetition were very similar to the networks found as a consequence of extensive training over onemonth period. The strength of the activity in these networks thus continued to increase/decrease with repeated training over a month.

\subsubsection{Coding of syllables through training of disyllabic pseudowords}

Is syllabic information extracted as a consequence of training on disyllabic pseudowords? To address the status of the Recombined stimuli (consisting of recombinations of the syllables from the Trained pseudowords), we investigated whether the activity related to the Recombined condition was influenced by repeated training of the pseudowords (and thus syllables) in the Trained condition. For this, we contrasted the Recombined and the Novel conditions on Day 28, and found greater activity for the Recombined condition in the left AG and right IPL. The reverse contrast (Novel > Recombined) revealed very similar areas as those observed in Novel $>$ Trained condition contrast. Note that there was no activity that was significantly higher for Trained $>$ Recombined condition on Day 28. However, the reverse contrast (Recombined $>$ Trained) showed areas that overlapped with Novel $>$ Trained condition (Table 2, Fig. 4 middle and bottom rows).

\section{Discussion}

The present data revealed that participants learned the mapping of Greek graphemes to Dutch phonemes quite rapidly, as accuracy performance for the Trained items reached almost ceiling level after 10 exposures on Day 1 . There was an additional benefit from repeated training over the course of one month, as illustrated by the reaction time reduction across the three test sessions. The Trained pseudowords were named much faster and more accurately than the Novel stimuli, made up from the same set of Greek graphemes. Thus, decoding clearly goes beyond grapheme-tophoneme mapping, resulting in the emergence of larger units of representation, such as "word" forms. Due to extensive exposure to a few Trained pseudowords throughout the experiment, participants could have developed an underspecified representation of the input, in terms of a few orthographic cues that distinguished a 
Table 2

Differences in activity on Day 28 .

\begin{tabular}{|c|c|c|c|c|c|c|c|c|}
\hline & \multicolumn{2}{|c|}{ Cluster } & \multicolumn{3}{|c|}{ Peak voxel } & \multicolumn{3}{|c|}{ MNI coordinates } \\
\hline & size & $P_{F W E}$ & $P_{F W E}$ & $\mathrm{~T}$ & $\mathrm{Z}$ & $\mathrm{X}$ & Y & $\mathrm{Z}$ \\
\hline \multicolumn{9}{|l|}{$\begin{array}{l}\text { Trained > Novel } \\
\text { (Voxel-level FWE corrected) }\end{array}$} \\
\hline left middle temporal gyrus & 125 & & 0.033 & 7.01 & 4.93 & -50 & -58 & 24 \\
\hline left angular gyrus & & & 0.01 & 7.79 & 5.22 & -54 & -56 & 32 \\
\hline left superior occipital gyrus & 56 & & 0.001 & 9.09 & 5.66 & -8 & -94 & 20 \\
\hline left inferior parietal lobule & 2 & & 0.032 & 7.04 & 4.94 & -54 & -54 & 48 \\
\hline right middle temporal gyrus & 33 & & 0.015 & 7.52 & 5.12 & 56 & -52 & 14 \\
\hline right supramarginal gyrus & 142 & & 0.003 & 8.53 & 5.48 & 60 & -34 & 30 \\
\hline right inferior parietal lobule & 6 & & 0.025 & 7.19 & 5 & 54 & -52 & 44 \\
\hline right angular gyrus & 11 & & 0.026 & 7.16 & 4.98 & 48 & -62 & 30 \\
\hline \multicolumn{9}{|l|}{$\begin{array}{l}\text { Novel > Trained } \\
\text { (Voxel-level FWE corrected) }\end{array}$} \\
\hline right middle occipital gyrus & 15 & & 0.011 & 7.71 & 5.19 & 32 & -82 & 36 \\
\hline right inferior occipital gyrus & 1946 & & 0 & 11.45 & 6.29 & 42 & -68 & -14 \\
\hline right inferior temporal gyrus & & & 0 & 12.47 & 6.52 & 48 & -64 & -6 \\
\hline right fusiform gyrus & & & 0 & 12.61 & 6.55 & 36 & -66 & -14 \\
\hline left inferior occipital gyrus & 1781 & & 0 & 12.13 & 6.45 & -36 & -70 & -10 \\
\hline left fusiform gyrus & & & 10 & 15.44 & 7.08 & -38 & -60 & -14 \\
\hline right superior occipital gyrus & 328 & & 0.013 & 7.61 & 5.16 & 30 & -66 & 40 \\
\hline right superior parietal lobule & & & 0 & 10.36 & 6.02 & 24 & -58 & 56 \\
\hline right postcentral gyrus & 86 & & 0.003 & 8.48 & 5.46 & 46 & -30 & 46 \\
\hline left superior parietal lobule & 844 & & 0 & 9.85 & 5.88 & -28 & -54 & 56 \\
\hline left inferior parietal lobule & & & 0 & 9.89 & 5.89 & -24 & -66 & 44 \\
\hline left precentral gyrus & 350 & & 0 & 10.91 & 6.16 & -54 & 4 & 34 \\
\hline left supplementary motor area & 74 & & 0.011 & 7.71 & 5.19 & -2 & 6 & 64 \\
\hline left superior frontal gyrus & 47 & & 0.008 & 7.9 & 5.26 & -24 & -4 & 56 \\
\hline right cerebellum & 74 & & 0.007 & 8.03 & 5.31 & -2 & -54 & -32 \\
\hline vermis & 6 & & 0.027 & 7.15 & 4.98 & 6 & -72 & -20 \\
\hline left cerebellum & 4 & & 0.034 & 7 & 4.92 & -22 & -38 & -42 \\
\hline \multicolumn{9}{|l|}{$\begin{array}{l}\text { Recombined > Novel } \\
\text { (Voxel-level FWE corrected) }\end{array}$} \\
\hline left angular gyrus & 6 & & 0.028 & 7.16 & 4.99 & -50 & -56 & 34 \\
\hline right inferior parietal lobule & 1 & & 0.038 & 6.97 & 4.91 & 50 & -58 & 28 \\
\hline right angular gyrus & 1 & & 0.045 & 6.86 & 4.87 & 48 & -60 & 30 \\
\hline \multicolumn{9}{|l|}{ (Cluster-size FWE corrected) } \\
\hline left angular gyrus & 1314 & 0 & & 7.16 & 4.99 & -50 & -56 & 34 \\
\hline left inferior parietal lobule & & & & 6.42 & 4.67 & -52 & -52 & 52 \\
\hline right angular gyrus & 1881 & 0 & & 6.97 & 4.91 & 50 & -58 & 28 \\
\hline right supramarginal gyrus & & & & 6.7 & 4.8 & 62 & -38 & 34 \\
\hline left precuneus & 1988 & 0 & & 6.4 & 4.67 & -4 & -58 & 26 \\
\hline left middle temporal gyrus & 490 & 0 & & 6.44 & 4.69 & -62 & -48 & -4 \\
\hline right Rolandic Operculum & 173 & 0.033 & & 6.03 & 4.5 & 58 & -4 & 8 \\
\hline \multicolumn{9}{|l|}{$\begin{array}{l}\text { Novel > Recombined } \\
\text { (Voxel-level FWE corrected) }\end{array}$} \\
\hline left inferior occipital gyrus & 32 & & 0.004 & 8.33 & 5.41 & -28 & -84 & -2 \\
\hline left middle occipital gyrus & 141 & & 0.001 & 9.19 & 5.69 & -32 & -92 & 14 \\
\hline left fusiform gyrus & 189 & & 0.001 & 9.52 & 5.79 & -36 & -62 & -10 \\
\hline left inferior occipital gyrus & & & 0.005 & 8.23 & 5.38 & -34 & -74 & -10 \\
\hline right inferior temporal gyrus & 146 & & 0.005 & 8.31 & 5.41 & 46 & -68 & -10 \\
\hline right inferior occipital gyrus & & & 0.005 & 8.31 & 5.41 & 44 & -66 & -14 \\
\hline right middle occipital gyrus & 28 & & 0.003 & 8.55 & 5.49 & 30 & -84 & 18 \\
\hline right fusiform gyrus & 24 & & 0.006 & 8.14 & 5.35 & 30 & -54 & -16 \\
\hline left superior parietal lobule & 133 & & 0 & 9.72 & 5.84 & -26 & -54 & 56 \\
\hline left inferior parietal lobule & 79 & & 0.001 & 9.6 & 5.81 & -38 & -40 & 46 \\
\hline left supramarginal gyrus & 2 & & 0.034 & 7.03 & 4.93 & -62 & -22 & 28 \\
\hline left precentral gyrus & 122 & & 0.004 & 8.35 & 5.42 & -54 & 4 & 34 \\
\hline right superior parietal lobule & 128 & & 0.001 & 9.26 & 5.71 & 28 & -50 & 58 \\
\hline right postcentral gyrus & 12 & & 0.009 & 7.88 & 5.26 & 42 & -32 & 50 \\
\hline right insula & 1 & & 0.043 & 6.9 & 4.88 & 34 & 28 & 0 \\
\hline
\end{tabular}

Trained > Recombined

(Voxel-level FWE corrected)

Recombined $>$ Novel

(Voxel-level FWE corrected) left middle occipital gyrus left inferior occipital gyrus left inferior temporal gyrus left fusiform gyrus right inferior temporal gyrus right fusiform gyrus

left inferior parietal lobule

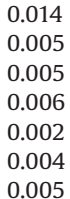

$$
\begin{array}{r}
-34 \\
-42 \\
-48 \\
-42 \\
50 \\
38 \\
-54
\end{array}
$$

$\begin{array}{rr}-90 & -4 \\ -76 & -10 \\ -66 & -10 \\ -52 & -16 \\ -66 & -6 \\ -68 & -18 \\ -30 & 46\end{array}$




\begin{tabular}{|c|c|c|c|c|c|c|c|c|}
\hline & \multicolumn{2}{|c|}{ Cluster } & \multicolumn{3}{|c|}{ Peak voxel } & \multicolumn{3}{|c|}{ MNI coordinates } \\
\hline & size & $P_{F W E}$ & $P_{F W E}$ & $\mathrm{~T}$ & $\mathrm{Z}$ & $\mathrm{X}$ & $\mathrm{Y}$ & $\mathrm{Z}$ \\
\hline left superior parietal lobule & 86 & & 0.011 & 7.71 & 5.2 & -24 & -64 & 44 \\
\hline left precentral gyrus & 139 & & 0.004 & 8.42 & 5.44 & -48 & 4 & 32 \\
\hline right superior occipital gyrus & 13 & & 0.016 & 7.47 & 5.11 & 30 & -66 & 40 \\
\hline right inferior parietal lobule & 60 & & 0.003 & 8.54 & 5.48 & 26 & -56 & 54 \\
\hline
\end{tabular}

FWE: family-wise error, MNI: Montreal Neurological Institute

specific trained pseudoword from the others, that triggered the response. If this were the case, Novel and Recombined pseudowords with similar letter combinations should have been mistaken for a Trained word. Errors, however, were not of this type for the Recombined and Novel pseudowords, but rather entailed a phoneme substitution or showed a disability to initiate the pronunciation within the short period of time. For this reason, it seems more likely that participants developed fully specified representations for the Trained pseudowords. Training not only induced the coding of pseudowords as a holistic word unit, as there was evidence for smaller units. The fact that the Recombined pseudowords were pronounced faster and more accurately than the Novel pseudowords suggests the emergence of novel syllabic units, since these were the units that were recombined.

Two distinct brain activity networks were observed. The first network comprised of visual areas, extending both ventrally to the inferior temporal cortex, and dorsally to the parietal cortex, and further to the left frontal gyrus. This network was dominant during the initial processing of the Trained pseudowords on Day 1, and for Novel pseudowords throughout the experiment (as displayed in the activation maps depicted in cold colors in Figs. 3-5). The second network consisted of the angular gyrus (AG), the supramarginal gyrus, the middle temporal gyrus (MTG), and the precuneus (as displayed in the activation maps depicted in warm colors in Figs. 3-5). This activity was largest for the Trained condition, increasing with stimulus repetitions during Day 1, and continued to increase over four weeks of repeated training. The Recombined condition activated both networks to a certain degree, but not to the full extent, as compared to the Novel condition (network 1) or to the Trained condition (network 2) (Figs. 3C and 4).

\subsection{Initial stage of symbol-to-sound conversion (network 1)}

It has been argued that in the initial stages of visual-word decoding, when the words are unfamiliar, we have to rely on the grapheme-to-phoneme conversion rules in order to articulate the printed letters - unless auditory information is provided (Coltheart, Rastle, Perry, Langdon, \& Ziegler, 2001; Ehri, 2005). In our experiment, we used Greek symbols to spell out new syllables that comply with Dutch syllable-structure rules. At the beginning of the experiment, participants were not very familiar with the correspondence between Dutch sounds and the Greek symbols, so we assumed orthography-phonology conversion to take place serially, at the level of small units, and phonological assembly to be effortful. This is reflected in the behavioral performance: accuracy was low when pressed for time (the Novel condition had the lowest accuracy scores, mainly due to a failure to pronounce within the limited time window, or to a sound mistake within the utterance). It also took long to name the Novel pseudowords throughout the experiment, but a continuous decrease in naming onset time was observed with repeated practice for the Trained condition.

On the neural level, the small steps of grapheme-to-phoneme conversions necessary for the correct speech output were accompanied by a particular network of activity in the brain (Figs. 3B and 4 right column). This network included bilateral inferior occipital cortex, extending to the fusiform gyrus ventrally. Successful letter-speech sound association is reflected by activation in the occipito-temporal cortex (Brem et al., 2010; Sandak et al., 2004; Xu et al., 2001). The activity in visual areas also extended dorsally and bilaterally, to the superior parietal lobe, extending to the inferior parietal lobe (IPL). These areas show increases in activity when processing highly demanding, visually complex material, or unfamiliar stimuli (Kravitz et al., 2011; Sandak et al., 2004), and for degraded word stimuli when serial reading strategies are deployed (Cohen et al., 2008). In line with our findings, a functional network comprising the posterior inferior temporal cortex and the parieto-occipital cortex was more engaged in an audio-visual matching task, when an auditory feature was associated with an unfamiliar visual input as compared to that of a familiar one (Hashimoto \& Sakai, 2004).

The frontal lobe is also known to activate when stimuli and task are demanding. Low-frequency words are known to activate the left inferior frontal junction, the inferior frontal gyrus (IFG), the anterior insula, the inferior parietal sulcus, and the subgenual cingulate, and bilateral supplementary motor areas (SMAs) (Graves et al., 2010). Planning of articulation is known to involve the anterior insula (Brown et al., 2009), and activity increases for unfamiliar speech sounds (Carreiras et al., 2006; Moser et al., 2009; Shuster, 2009). Indeed, we observed that these areas were more active for the Novel condition.

In sum, we identified a network of brain regions when less familiar visual input needs to be decoded. These regions corresponded to areas in which visual input is processed in ventral (in the inferior temporal lobes) and dorsal streams (in the parietal lobes) (Blomert, 2011), and then translated into motor programs for correct articulation (in the insula, pars opercularis of the left IFG, and the pre- and post-central gyri). All these processes are less automated for the Novel than for the Trained pseudowords, resulting in higher demands during each of the processes. This leads to activity increase in areas dedicated to visual processing, phonological assembly, and preparation of articulation. These areas showed a decrease in activity for the Trained condition with repeated exposure on Day 1, and, consistently, also after a month of repeated training. It is plausible to assume that, with practice, decoding and articulatory preparation became more automated such that attention-related processes were less required.

\subsection{Stabilization of new pseudoword decoding through extensive training (network 2)}

After repeated exposure, participants were able to pronounce the Trained pseudowords more automatically and efficiently. This is reflected in the reaction time decrease for the Trained pseudowords, over the course of one month. On the neural level, an increase in brain activity related to efficient conversion of print to sound was found in the bilateral AG, the bilateral precuneus extending to the middle cingulate cortex, and the bilateral MTG. 
Trained $>$ Novel

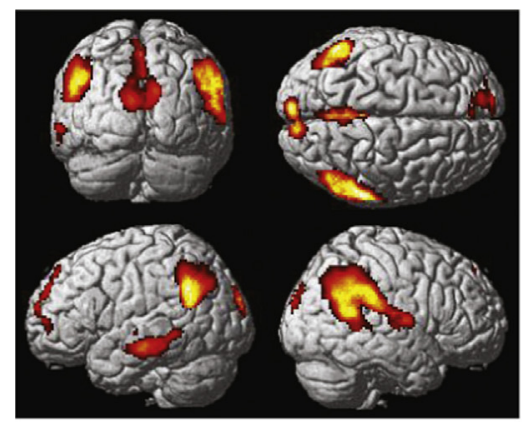

Recombined $>$ Novel

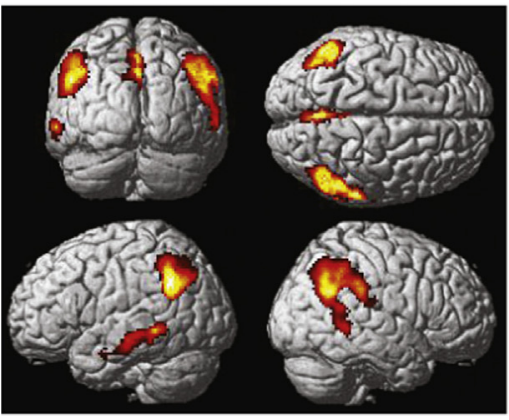

Trained $>$ Recombined

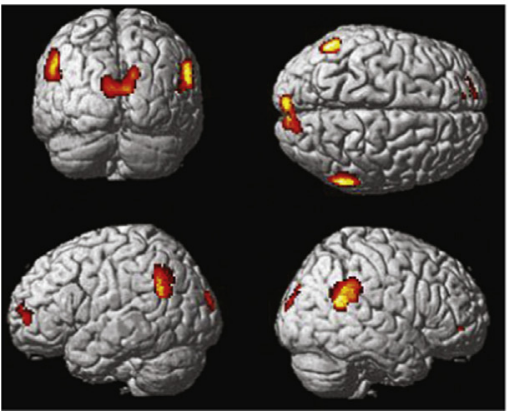

Novel > Trained
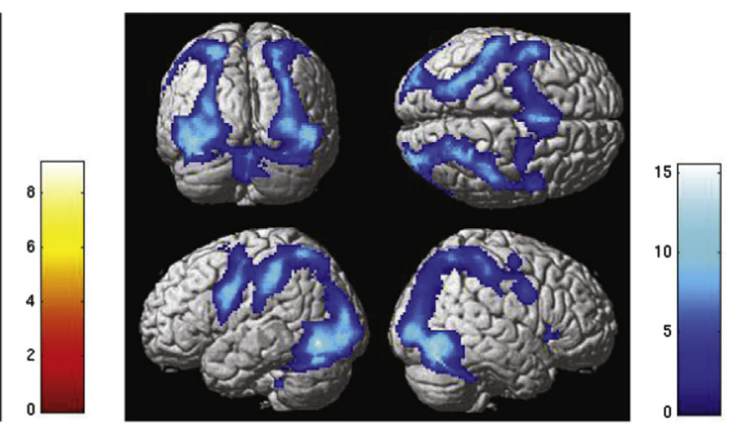

Novel > Recombined
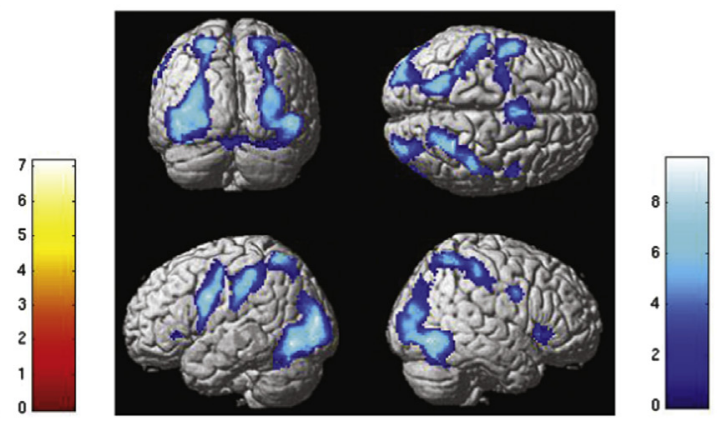

Recombined > Trained
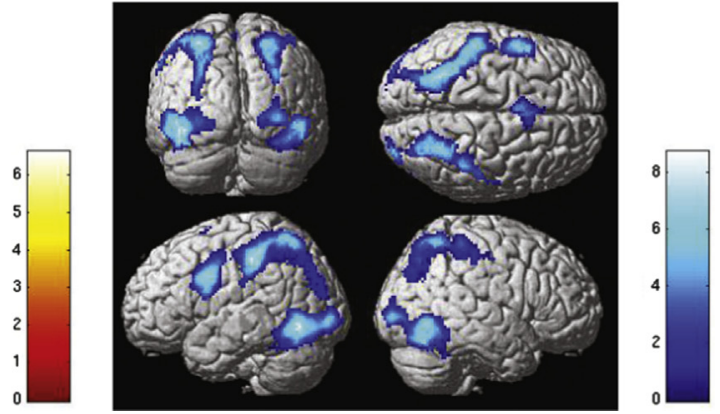

Fig. 4. Activity difference between conditions on Day 28. For illustration purposes, the figures are thresholded at voxel $p<.001$, cluster-level corrected $\left(P_{F W E}<.05\right)$ on the whole brain. (For interpretation of the references to color in this figure, the reader is referred to the web version of this article.)

A

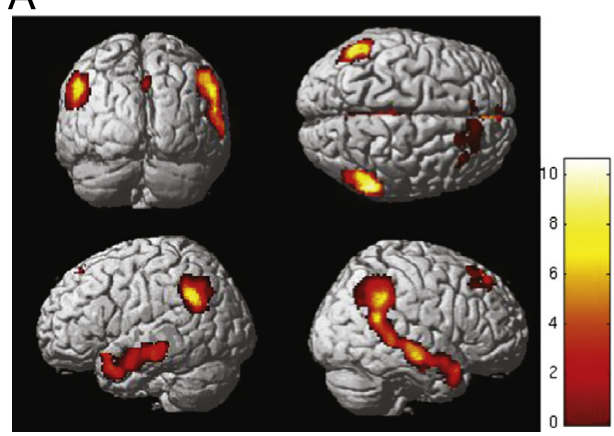

B

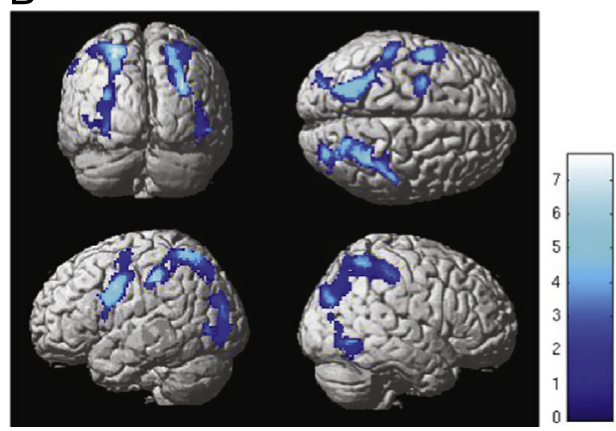



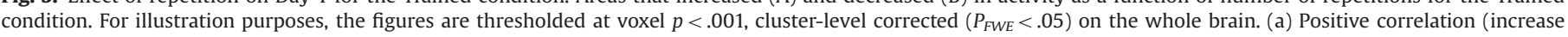
with repetition) and (b) Negative correlation (decrease with repetition).

One way of interpreting our data is that a unitary code for the conversion from script-to-sound for the stimulus as a whole emerged with repeated training, and that this representation is stored in the network described above.
When we sound out visual inputs, regions that process orthography, phonology, as well as motor-programming areas must exchange information. Many neuroimaging studies so far have provided evidence for the neural correlates of reading, which 
Table 3

Day 1 change with repetition for Trained condition.

\begin{tabular}{|c|c|c|c|c|c|c|c|c|}
\hline & \multicolumn{2}{|c|}{ Cluster } & \multicolumn{3}{|c|}{ Peak voxel } & \multicolumn{3}{|c|}{ MNI coordinates } \\
\hline & size & $P_{F W E}$ & $P_{F W E}$ & $\mathrm{~T}$ & Z & $\mathrm{X}$ & $\mathrm{Y}$ & Z \\
\hline \multicolumn{9}{|l|}{$\begin{array}{l}\text { Increase in activity with repetition } \\
\text { (Voxel-level FWE corrected) }\end{array}$} \\
\hline left inferior parietal lobe & 74 & & 0.003 & 8.64 & 5.52 & -52 & -54 & 40 \\
\hline left angular gyrus & & & 0.008 & 8.06 & 5.32 & -56 & -60 & 30 \\
\hline right inferior parietal lobe & 4 & & 0.025 & 7.37 & 5.07 & 56 & -48 & 48 \\
\hline right angular gyrus & 33 & & 0.022 & 7.43 & 5.09 & 58 & -50 & 36 \\
\hline right supramarginal gyrus & & & 0.009 & 7.99 & 5.3 & 60 & -46 & 36 \\
\hline right middle temporal gyrus & 37 & & 0 & 10.6 & 6.08 & 58 & -52 & 18 \\
\hline right middle temporal gyrus & 24 & & 0.016 & 7.65 & 5.17 & 64 & -16 & -14 \\
\hline \multicolumn{9}{|l|}{ (Cluster-size FWE corrected) } \\
\hline right middle temporal gyrus & 3166 & 0 & & 10.6 & 6.08 & 58 & -52 & 18 \\
\hline right supramarginal gyrus & & & & 7.99 & 5.3 & 60 & -46 & 36 \\
\hline right middle temporal gyrus & & & & 7.65 & 5.17 & 64 & -16 & -14 \\
\hline left inferior parietal lobe & 851 & 0 & & 8.64 & 5.52 & -52 & -54 & 40 \\
\hline left angular gyrus & & & & 8.06 & 5.32 & -56 & -60 & 30 \\
\hline left supramarginal gyrus & & & & 6.62 & 4.76 & -60 & -52 & 32 \\
\hline left middle temporal gyrus pole & 1127 & 0 & & 8.21 & 5.37 & -46 & 10 & -30 \\
\hline left middle temporal gyrus & & & & 6.11 & 4.54 & -56 & -4 & -20 \\
\hline left inferior temporal gyrus & & & & 4.79 & 3.87 & -52 & -30 & -16 \\
\hline left superior temporal gyrus & & & & 4.51 & 3.7 & -48 & -8 & -12 \\
\hline left middle cingulate cortex & 1451 & 0 & & 7.02 & 4.93 & -6 & -40 & 40 \\
\hline right precuneus & & & & 5.68 & 4.33 & 6 & -58 & 40 \\
\hline right posterior cingulate cortex & & & & 4.59 & 3.75 & 2 & -46 & 28 \\
\hline left anterior cingulate cortex & 1085 & 0 & & 6.23 & 4.59 & -6 & 50 & 16 \\
\hline right anterior cingulate cortex & & & & 5.71 & 4.35 & 6 & 48 & 12 \\
\hline right superior frontal gyrus & 232 & 0 & & 5.64 & 4.31 & 22 & 28 & 56 \\
\hline right superior frontal gyrus & & & & 5.44 & 4.22 & 16 & 40 & 50 \\
\hline right superior medial frontal gyrus & & & & 5.28 & 4.13 & 8 & 36 & 56 \\
\hline \multicolumn{9}{|l|}{$\begin{array}{l}\text { Decrease in activity with repetition } \\
\text { (Voxel-level FWE corrected) }\end{array}$} \\
\hline left inferior parietal lobe & 9 & & 0.014 & 7.74 & 5.2 & -36 & -36 & 42 \\
\hline left precentral gyrus & 7 & & 0.019 & 7.54 & 5.13 & -50 & -4 & 42 \\
\hline \multicolumn{9}{|l|}{ (Cluster-size FWE corrected) } \\
\hline left inferior parietal lobe & 1750 & 0 & & 7.74 & 5.2 & -36 & -36 & 42 \\
\hline left superior parietal lobe & & & & 6.7 & 4.8 & -24 & -58 & 60 \\
\hline left precentral gyrus & 853 & 0 & & 7.54 & 5.13 & -50 & -4 & 42 \\
\hline left inferior frontal gyrus (pars opercularis) & & & & 5.44 & 4.22 & -54 & 10 & 26 \\
\hline right superior occipital gyrus & 1943 & 0 & & 6.52 & 4.72 & 22 & -60 & 48 \\
\hline right postcentral gyrus & & & & 6.45 & 4.69 & 32 & -36 & 48 \\
\hline right middle occipital gyrus & & & & 6.35 & 4.65 & 32 & -80 & 28 \\
\hline left superior frontal gyrus & 252 & 0 & & 5.76 & 4.37 & -22 & -8 & 60 \\
\hline left middle frontal gyrus & & & & 5.64 & 4.31 & -30 & -2 & 52 \\
\hline right inferior temporal gyrus & 330 & 0 & & 5.66 & 4.32 & 42 & -60 & -10 \\
\hline right middle temporal gyrus & & & & 4.25 & 3.55 & 48 & -72 & 6 \\
\hline left inferior occipital gyrus & 441 & 0 & & 5.14 & 4.06 & -32 & -82 & -6 \\
\hline left middle occipital gyrus & & & & 4.68 & 3.8 & -28 & -82 & 26 \\
\hline
\end{tabular}

FWE: family-wise error, MNI: Montreal Neurological Institute

includes areas related to visual and auditory processing, and to motor preparation, but also areas concerned with semantic representations (Jobard, Crivello, \& Tzourio-Mazoyer, 2003; Price, 2010; Taylor, Rastle, \& Davis, 2013). For the processing of visual input, the left occipito-temporal area, also known as "visual word form area" (VWFA), is known to code recurring combinations of letters as integrated visual perceptual units for rapid and effortless reading (McCandliss et al., 2003), although some studies also assign associated phonological coding to this area (Sandak et al., 2004; Xu et al., 2001). Phonological processing is reflected as activity in the mid to posterior superior temporal sulcus (STS) (Hickok \& Poeppel, 2007). Linking of visual and auditory information recruits areas in the junction of temporal and parietal cortex, for both words and pseudowords (Demonet, Price, Wise, \& Frackowiak, 1994; Price, Green, \& von Studnitz, 1999). The MTG seems to code for phonological memory representations of known words (Hagoort et al., 1999), and is often referred to as convergence zone that combines multimodal information (for a review: Binder \& Desai, 2011). The above findings suggest that memory for orthography resides in occipito-temporal cortex, whereas memory of phonology is coded in the superior part of the temporal cortex. The MTG and the temporo-parietal junction seem to process information about specific orthographic-phonological associations. The functions of the above-mentioned areas in the left hemisphere fit with the theory put forward by Pugh et al. (2000), who consider the ventral occipito-temporal area as a memorybased word identification system, and the temporo-parietal circuit as involved in the extraction of the relationship between orthography, phonological form, morphological and lexical-semantic information, resulting in integrated representations.

The AG and the MTG are known to be activated more for familiar words compared to unfamiliar letter strings. They are also reported to be more active when reading irregular words. The activity is thought to reflect the use of a mental lexicon, a node that connects different 
aspects of the word (e.g. orthography, phonological form, morphological and lexical-semantic information), when reading these words (Binder et al., 2003). Since our trained pseudowords were meaningless, the activity increases in the AG, the MTG, and the precuneus could not be influenced by access to semantic information. We would rather argue that they code for orthography-phonology association of the novel words. This multimodal representation emerged through repeated training on articulating these pseudowords. The representation of the novel words probably involved a holistic unit after multiple repetitions, which in turn resulted in higher accuracy and speed after a month of training. Interestingly, not only the whole word unit but also sub-units (in our case the syllables) seem to have emerged. We will discuss this in Section 4.3.

There was a general increase over time in the AG and precuneus, which was present in both Trained and Novel conditions, but the increase over time was larger for the Trained than that for the Novel condition. A general increase related to the task would imply that the AG and the precuneus are involved in coding the conversion of Greek script into Dutch phonemes on a grapheme-by-grapheme basis, a process that is shared by the two conditions. However, because the Trained and Recombined conditions both showed greater activity than the Novel condition in the bilateral AG and the left MTG on Day 28, these areas might also be coding larger, chunked memory representations, such as syllables and whole-word units, which would lead to a more efficient decoding and articulation for the Trained as well as for the Recombined pseudowords.

One caveat is that areas such as the precuneus, the medial, lateral and inferior parietal cortex, and the medial prefrontal cortex that partially overlap with our results, are known to increase in activity when the brain is at a resting state, known as the Default Mode Network (Mason et al., 2007; Raichle \& Snyder, 2007). In fMRI studies, easy tasks tend to show a relative increase in these areas when compared with perceptually challenging tasks. It is true that with extensive training, our participants became very efficient at articulating the pseudowords of the Trained condition, rendering this condition into an easy one compared to the Novel and the Recombined conditions. On the other hand, the network that shows an increase in activity for memory retrieval is very similar to the default mode network (Schacter, Addis, \& Buckner, 2007). Therefore, the activation increase in the AG and the left MTG might also reflect access to emergent memory representations for the stimuli of the Trained condition, and to emergent novel syllables for the Recombined condition (see Section 4.3 for discussion on syllable units).

We did not observe increased activity with repeated practice in the VWFA in the fusiform gyrus, an area known to code orthography (Braet, Wagemans, \& Op de Beeck, 2012; McCandliss et al., 2003). This area has been reported to sharpen and consequently reduce in activation when participants become more skilled at reading (Dehaene et al., 2010; Mochizuki-Kawai, Tsukiura, Mochizuki, \& Kawamura, 2006). Another possible reason for the lack of VWFA activation in the Trained condition might be the multiple repetition of Trained stimuli. Firing of neurons that represent incoming information tends to reduce when the same input information is processed repeatedly (van Turennout, Bielamowicz, \& Martin, 2003). This is termed repetition suppression, or adaptation (Grill-Spector, Henson, \& Martin, 2006). Since the pseudowords from the Trained condition were repeated 10 times each over the course of each fMRI session (and many times during the training sessions), average activity for the Trained condition might well have been lower than in the Recombined or the Novel condition, due to repetition suppression.

It is true that interpretation of the activation differences between the Trained and the other two conditions should be interpreted with care, as Trained condition trials contained stimuli that were repeated (it was unavoidable for the fMRI data due to a very small number of trained items), whereas there were no repetitions of the exact same stimulus in the other conditions. Indeed when we contrasted the activity increase/decrease with repetition of the Trained condition on Day 1, we observed similar patterns of activation. One interpretation is that this network reflects repetition, general habituation, or less novelty-related attention. As such, the network would not bear on the emergence of new pseudoword/syllable representations. But it is important to note the high degree of similarity between activation patterns observed for the contrasts for which repetition within the session is constant. One is the contrast between the Recombined and the Novel condition, with no repetition of the same stimuli within a run. The other contrast concerns the Trained condition compared across the sessions, where all sessions have an equal number of repetitions. Both contrasts showed the same two networks; one that is prominent when compilation of smaller units is required for pronunciation, thus necessitating multiple steps of processing (network 1, the Novel condition, initial phase of the Trained condition, and to a lesser degree the Recombined condition), and a more efficient one that can benefit from chunking, resulting in the emergence of syllabic or word units (network 2, the Trained condition with more repetition and training, and the Recombined condition compared to the Novel condition). For these reasons, we believe that our effects are not merely due to the presence or absence of repetition in the different conditions but rather to the processing change due to emergence of word/syllable representations.

\subsection{Role of syllables in new pseudoword decoding}

Although the pseudowords from the Recombined condition were never trained, and the specific combinations of the disyllables appeared only once during the fMRI and Behavioral test sessions on Day 5 and Day 28, participants were more accurate and faster to pronounce the Recombined pseudowords compared to pseudowords in the Novel condition. This implies that there is flexibility in the learning system that allows syllabic units to be extracted from the trained pseudowords and stored as a result of repeated practice with these trained pseudowords. These same syllables constituted the pseudowords in the Recombined condition, and facilitated their processing. We observed better and faster performance in pronouncing the Recombined words as compared to the Novel words. We interpreted this difference as evidence that the participants were able to make use of novel, stored syllabic units, derived from the syllables of the trained pseudowords. The Trained and Recombined pseudowords showed activation increases in the same areas, namely the AG and the MTG. This suggests that the AG and the MTG are coding for integrated orthography-phonology conversion, and they seem to do so in multiple levels of units (from small grapheme-phoneme conversion to larger units entailing syllables and holistic word units), as long as they are occurring frequently. To confirm that syllabic units were extracted and coded when trained on multisyllabic pseudowords, one could test whether pronouncing single syllables (that were present in the Trained condition) involved the same areas of the brain as pronouncing Trained pseudowords. With the current setup, we could not strictly dissociate between the interpretations that the emergence of syllabic units occurred naturally through the repeated training of disyllabic pseudowords, or that it was a conscious realization on the participants' side after they had gone through the fMRI test on Day 5 when they were confronted with recombined syllables for the first time. Their experience of the Recombined condition tested on Day 5 might have influenced their response on Day 28 because they were now aware of the possibility of the recombined syllables. However, this interpretation seems unlikely given that accuracy and reaction time response for the Recombined condition did not improve from Day 5 to Day 28.

A limitation to our findings is that even though our focus was on the syllables for sub-word level units, we cannot rule out the 
possibility that the emergence of (Greek) orthography to (Dutch) phonology conversion coding is occurring on even smaller units such as bi- and trigrams, as these were also repeated more for the Recombined condition than for the Novel condition. It was, however, impossible to keep the frequencies of occurrence for bi- and trigrams equal for the Recombined and Novel conditions, given the nature of the material used here.

Even though naming of pseudowords in the Recombined condition was faster than those from the Novel condition, it was still slower than that of the Trained condition. On the neural level, the Recombined stimuli involved more activity in the visual-attention network and the left precentral area than the Trained stimuli, indicating that naming recombined syllables required additional processing compared to the Trained condition. This activity pattern is similar to that of the contrast between Novel and Trained conditions (network 1 in blue shade Fig. 4 right column). Since the specific combinations of the syllables used in the Recombined condition were never trained, additional visual processing, working memory, and motor preparation were necessary to assemble the syllables for the articulation of the Recombined condition. The increased activity for the Recombined compared to the Trained condition may reflect extra phonological assembly process on the syllabic level.

\subsection{Possible roles for the areas observed in our data}

What is the neural network for decoding unfamiliar and familiar orthography? Although our data do not provide information on the temporal processing flow from visual input to speech output, it is plausible to assume that both feed-forward and feed-backward processes are involved (Price \& Devlin, 2011). Our brain can identify a word as a word at a speed that is much faster than recognizing it letter by letter from left to right, and then as a whole after reaching the last letter (Nazir et al., 1998). The same holds for articulation (Levelt, 2001). Frequently occurring units are probably coded and stored in chunks or larger units than graphemes and phonemes, such that when reading aloud familiar words, many compilation steps needed for assembling decoded segments can be skipped. Triggered by the visual input, two processes can work in parallel: one that decodes serially from left to right, and another that scans for a possible match in the existing memory representations, as large and holistic as possible, both in orthography and phonology. Processing below the word level results in smaller units, such as syllables or groups of letters that co-occur often enough to have a stored representation. The smaller the units of analysis are, the more is the need for phonological assembly of each piece of retrieved information, resulting in a longer and more effortful process.

From our findings, one can speculate about the specific areas involved in the above processes. When visual input is presented, information is processed along the visual stream in the occipital cortex to the ventral part of the temporal cortex. The left fusiform gyrus is known to code familiar letter strings (McCandliss et al., 2003), and can also react to familiarized consonant strings (Fisher, Cortes, Griego, \& Tagamets, 2011). The dorsal visual stream extending to the superior parietal lobe is known to activate when visuo-spatial processing demands are high (Corbetta \& Shulman, 2002; Galletti, Kutz, Gamberini, Breveglieri, \& Fattori, 2003). For unfamiliar letter-string combinations (Novel condition), visual processing was more demanding probably due to the fact that the letter strings had to be decoded in a letter-by-letter fashion. Furthermore, for the Novel condition without holistic representations, the retrieved sounds associated to each symbol had to be kept online in working memory, to assemble the phonemes for articulation, a demand posed upon the PFC (Smith, Jonides, Marshuetz, \& Koeppe, 1998). This would cause more activation of the IFG for the Novel than the Trained pseudowords (for example Joubert et al., 2004), which is in line with our finding. In contrast, chunked information (holistic word representation) could have emerged as a result of training, and possibly coded in the AG, the precuneus and the MTG, leading to increased activity in these areas for reading out the coded memory representations when preparing to articulate. With holistic representations, less or no processing is needed for the assembly of smaller units, resulting in a fast and efficient articulation. Furthermore, smaller units derived from a longer word, namely its syllables, would become represented in similar brain structures, such that articulation of recombined syllables benefited from repeated training of novel disyllabic words.

\section{Conclusion}

In this experiment, we showed that while unfamiliar Dutchlike pseudowords written in Greek script can be sounded out if given enough time, their pronunciation becomes more and more fluent with repeated practice of articulation. This is probably due to a shift from a grapheme-to-phoneme decoding to a more holistic conversion, resulting in efficient articulation. Initially, smaller units are decoded, involving increased activity in the visual-attention areas of the occipito-temporal and occipitoparietal cortices, increased working-memory processing in the frontal cortex, and motor planning and coordination in the precentral gyrus and the anterior insula. Once memory representations of chunked information are established in areas encompassing the angular gyrus, the precuneus and the middle temporal gyrus, the articulation becomes more fluent and less effortful. The two networks are flexible and interactive such that recurring units - such as syllables - are extracted and coded in the latter network, and can be retrieved and recombined for articulation, even when these units are newly combined.

\section{Acknowledgments}

The authors would like to acknowledge Jos Olders for collection of a part of the data, Paul Gaalman for technical help, and all the participants for attending without fail all sessions throughout a whole month. This work was supported by a grant from the Netherlands Organization for Scientific Research (NWO, 016.005.061 and 056-33014).

\section{Appendix A}

See Tables A.1 and A.2.

Table A.1

Used alphabets and their conversion to Greek orthography.

\begin{tabular}{|c|c|c|c|c|c|c|c|c|c|c|c|c|c|c|c|c|c|c|c|c|}
\hline \multicolumn{21}{|c|}{ Alphabet } \\
\hline Latin & $\mathrm{a}$ & $\mathrm{b}$ & e & $\mathrm{f}$ & $\mathrm{g}$ & $\mathrm{i}$ & $\mathrm{j}$ & $\mathrm{k}$ & 1 & $\mathrm{~m}$ & $\mathrm{n}$ & 0 & oo & $\mathrm{p}$ & $r$ & $\mathrm{~s}$ & $\mathrm{t}$ & uu & $\mathrm{v}$ & $\mathrm{w}$ \\
\hline Greek & $\alpha$ & $\beta$ & $\varepsilon$ & $\varphi$ & $\chi$ & I & I & $\kappa$ & $\lambda$ & $\mu$ & $\nu$ & 0 & $\omega$ & $\pi$ & $\rho$ & $\sigma$ & $\tau$ & 0 & $>$ & $\sim$ \\
\hline
\end{tabular}


Table A.2

List of pseudowords in Greek alphabet and their pronunciations.

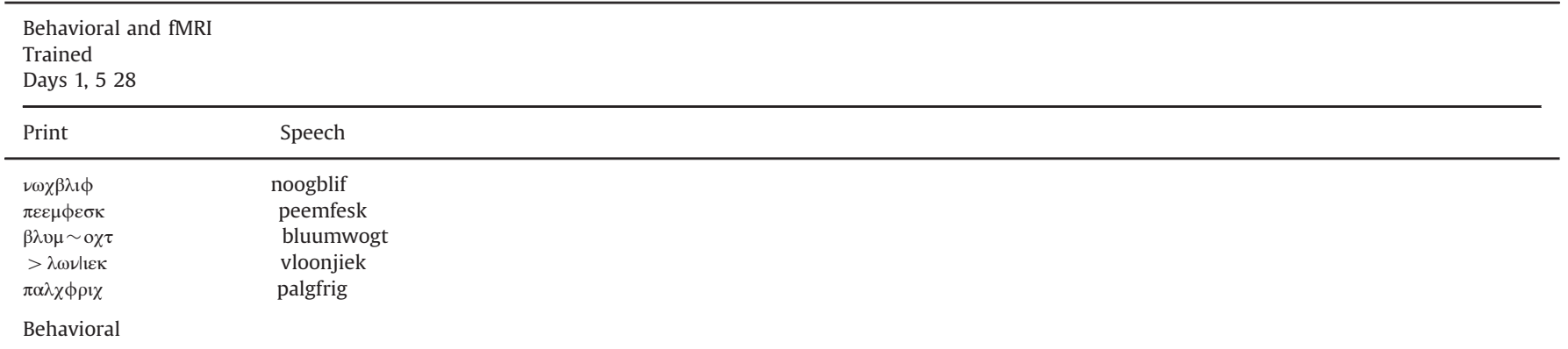

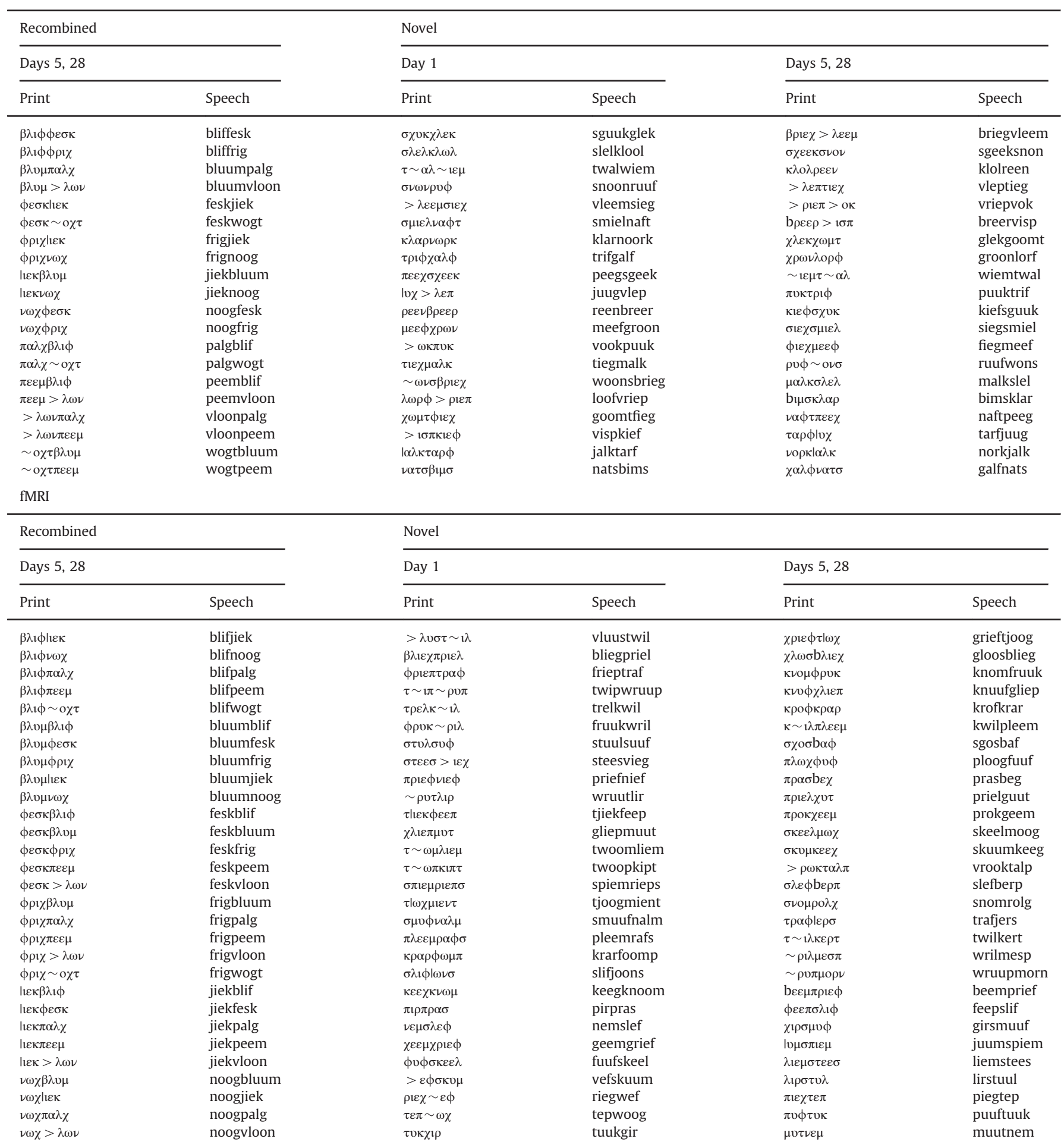


Table A.2 (continued)

\begin{tabular}{|c|c|c|c|c|c|}
\hline$\nu \omega \chi \sim \mathrm{o} \chi \tau$ & noogwogt & $\rho \iota \rho \pi \cup \phi$ & rirpuuf & $\nu 1 \varepsilon \phi \pi ı$ & niefpir \\
\hline$\pi \alpha \lambda x \beta \lambda \nu \mu$ & palgbluum & $\beta \varepsilon \chi \beta \varepsilon \mu \sigma$ & begbems & $\sigma \alpha \alpha \phi \chi \varepsilon \rho \nu$ & saafgern \\
\hline$\pi \alpha \lambda \chi \phi \varepsilon \sigma \kappa$ & palgfesk & $\chi v \tau \kappa \omega \rho \kappa$ & guutkoork & $\sim o \chi \sigma 1 \varepsilon \rho \phi$ & wogsierf \\
\hline$\left.\pi \alpha \lambda \chi\right|_{1 \varepsilon \kappa}$ & palgjiek & $\beta \alpha \phi \nu \mu \sigma$ & bafnims & $\sigma \nu \phi \sigma \varepsilon \mu \sigma$ & suufsems \\
\hline$\pi \alpha \lambda \chi \pi \varepsilon \varepsilon \mu$ & palgpeem & $\sigma \varepsilon \varepsilon \kappa>\varepsilon \varepsilon \mu \pi$ & seekveemp & $>1 \varepsilon \chi \tau \sim \omega \mu$ & viegtwoom \\
\hline$\pi \alpha \lambda \chi>\lambda \omega \nu$ & palgvloon & $\mu \omega \chi \pi \alpha \kappa \sigma$ & moogpaks & $\sim \varepsilon \phi \sigma \iota \rho \phi$ & wefsirf \\
\hline$\pi \varepsilon \varepsilon \mu \beta \lambda \nu \mu$ & peembluum & $\sigma \varepsilon \mu \sigma>\rho \omega \kappa$ & semsvrook & 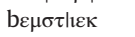 & bemstjiek \\
\hline$\pi \varepsilon \varepsilon \mu \phi \rho \imath \chi$ & peemfrig & $\mid \varepsilon \rho \sigma \kappa \nu \nu \phi$ & jersknuuf & $\kappa ı \pi \tau \lambda \varepsilon \sigma \kappa \kappa$ & kiptlesk \\
\hline$\pi \varepsilon \varepsilon \mu \mid \imath \kappa \kappa$ & peemjiek & $\kappa \varepsilon \rho \tau \sigma \chi \omega \sigma$ & kertsgoos & b $\varepsilon \varepsilon \mu \sigma \phi \rho 1 \varepsilon \pi$ & beemsfriep \\
\hline$\pi \varepsilon \varepsilon \mu \nu \omega \chi$ & peemnoog & $\tau \nu \rho \phi \sigma \nu \omega \mu$ & tuurfsnoom & $\phi о \mu \pi \tau \rho \varepsilon \lambda$ & fomptrel \\
\hline$\pi \varepsilon \varepsilon \mu \sim \mathrm{o} \chi \tau$ & peemwogt & $\sigma ı \rho \phi \kappa \rho \omega \phi$ & sierfkroof & $\mid \omega \nu \sigma \tau \sim 1 \pi$ & joonstwip \\
\hline$>\lambda \omega \nu \beta \lambda_{I} \phi$ & vloonblif & $\chi \varepsilon \rho \nu \pi \rho \omega \kappa$ & gernprook & $\kappa о \rho \kappa \tau \sim \omega \pi$ & korktwoop \\
\hline$>\lambda \omega \nu \phi \varepsilon \sigma \kappa$ & vloonfesk & $\tau_{1} \phi \tau \pi \lambda \omega \chi$ & tiftploog & $\mu 1 \varepsilon \nu \tau>\lambda \nu \sigma$ & mientvluus \\
\hline$>\lambda \omega \nu \phi \rho \iota \chi$ & vloonfrig & $\sigma \iota \rho \phi \chi \lambda \omega \sigma$ & sirfgloos & $\nu \alpha \lambda \mu \sim \rho v \tau$ & nalmwruut \\
\hline$>\lambda \omega \nu \nu \omega \chi$ & vloonnoog & $\mu \varepsilon \sigma \pi \mid v \mu$ & mespjuum & $\nu ц \mu \sigma \sigma \varepsilon \varepsilon \kappa$ & nimsseek \\
\hline$>\lambda \omega \nu \sim \mathrm{o} \chi \tau$ & vloonwogt & $\lambda \varepsilon \sigma \kappa \sigma \alpha \alpha \phi$ & lesksaaf & $\pi \alpha \kappa \sigma \rho 1 \varepsilon \chi$ & paksrieg \\
\hline$\sim \mathrm{o} \chi \tau \beta \lambda_{1} \phi$ & wogtblif & $\tau \alpha \lambda \pi \beta \varepsilon \varepsilon \mu$ & talpbeem & $\rho \alpha \phi \sigma \rho \iota \rho$ & rafsrir \\
\hline$\sim \mathrm{o} \chi \tau \phi \varepsilon \sigma \kappa$ & wogtfesk & $\mu \omega \rho \nu \pi \mid \varepsilon \chi$ & moornpieg & $\rho 1 \varepsilon \pi \sigma>\varepsilon \phi$ & riepsvef \\
\hline$\sim \mathrm{o} \chi \tau \phi \rho \mathrm{l} \chi$ & wogtfrig & $>\varepsilon \mu \pi>1 \rho \mu$ & vempvirm & $>\varepsilon \varepsilon \mu \pi \tau ו \phi \tau$ & veemptift \\
\hline$\sim o \chi \tau \nu \omega \chi$ & wogtnoog & $\rho \omega \lambda \chi \sim \omega \tau \sigma$ & roolgwoots & $>1 \rho \mu \tau v \rho \phi$ & virmtuurf \\
\hline$\sim \mathrm{o} \chi \tau \pi \alpha \lambda \chi$ & wogtpalg & $\beta \varepsilon \rho \pi \beta \varepsilon \varepsilon \mu \sigma$ & berpbeems & $\sim \mathrm{o} \tau \sigma>\varepsilon \mu \pi$ & wotsvemp \\
\hline
\end{tabular}

\section{References}

Ans, B., Carbonnel, S., \& Valdois, S. (1998). A connectionist multiple-trace memory model for polysyllabic word reading. Psychological Review, 105(4), $678-723$.

Barlow, H. B. (1989). Unsupervised learning. Neural Computation, 1(3), 295-311.

Binder, J. R., \& Desai, R. H. (2011). The neurobiology of semantic memory. Trends in Cognitive Science, 15(11), 527-536.

Binder, J. R., McKiernan, K. A., Parsons, M. E., Westbury, C. F., Possing, E. T., Kaufman, J. N., et al. (2003). Neural correlates of lexical access during visual word recognition. Journal of Cognitive Neuroscience, 15(3), 372-393.

Blomert, L. (2011). The neural signature of orthographic-phonological binding in successful and failing reading development. Neuroimage, 57(3), 695-703.

Braet, W., Wagemans, J., \& Op de Beeck, H. P. (2012). The visual word form area is organized according to orthography. Neuroimage, 59(3), 2751-2759.

Brem, S., Bach, S., Kucian, K., Guttorm, T. K., Martin, E., Lyytinen, H., et al. (2010) Brain sensitivity to print emerges when children learn letter-speech sound correspondences. Proceedings of the National Academy of Sciences of the United States of America, 107(17), 7939-7944.

Brett, M., Penny, W., \& Kiebel, S. (2004). Introduction to random field theory. In: R. S. J. Frackowiak, K. J. Friston, C. D. Frith, R. J. Dolan, C. J. Price, S. Zeki, J. T. Ashburner, \& W. D. Penny (Eds.), Human brain function (2nd ed.). Burlington: Academic Press (Chapter 44)

Brown, S., Laird, A. R., Pfordresher, P. Q., Thelen, S. M., Turkeltaub, P., \& Liotti, M (2009). The somatotopy of speech: Phonation and articulation in the human motor cortex. Brain and Cognition, 70(1), 31-41.

Carreiras, M., Mechelli, A., Estévez, A., \& Price, C. J. (2007). Brain activation for lexical decision and reading aloud: Two sides of the same coin? Journal of Cognitive Neuroscience, 19(3), 433-444.

Carreiras, M., Mechelli, A., \& Price, C. J. (2006). Effect of word and syllable frequency on activation during lexical decision and reading aloud. Human Brain Mapping 27(12), 963-972.

Carreiras, M., Riba, J., Vergara, M., Heldmann, M., \& Münte, T. F. (2009). Syllable congruency and word frequency effects on brain activation. Human Brain Mapping, 30(9), 3079-3088.

Cohen, L., Dehaene, S., Vinckier, F., Jobert, A., \& Montavont, A. (2008). Reading normal and degraded words: Contribution of the dorsal and ventral visual pathways. Neuroimage, 40(1), 353-366.

Coltheart, M. (2005). Modeling reading: the dual-route approach. In: M. Snowling \& C. Hulme (Eds.), The science of reading: a handbook (pp. 6-23). Oxford: Blackwell.

Coltheart, M., Rastle, K., Perry, C., Langdon, R., \& Ziegler, J. (2001). Drc: A dual route cascaded model of visual word recognition and reading aloud. Psychological Review, 108(1), 204-256.

Corbetta, M., \& Shulman, G. L. (2002). Control of goal-directed and stimulus-driven attention in the brain. Nature Reviews Neuroscience, 3(3), 201-215.

Dehaene, S., Cohen, L., Sigman, M., \& Vinckier, F. (2005). The neural code for written words: a proposal. Trends in Cognitive Sciences, 9(7), 335-341.

Dehaene, S., Pegado, F., Braga, L. W., Ventura, P., Filho, G. N., Jobert, A., et al. (2010) How learning to read changes the cortical networks for vision and language. Science, 330(6009), 1359-1364.

Demonet, J. F., Price, C., Wise, R., \& Frackowiak, R. S. (1994). A pet study of cognitive strategies in normal subjects during language tasks. Influence of phonetic ambiguity and sequence processing on phoneme monitoring. Brain, 117(Pt 4) 671-682.

Ehri, L. C. (2005). Development of sight word reading: Phases and findings. In: M. Snowling, \& C. Hulme (Eds.), The science of reading, a handbook (pp. 135-154). Oxford: Blackwell Publishing.
Fisher, J. E., Cortes, C. R., Griego, J. A., \& Tagamets, M. A. (2011). Repetition of letter strings leads to activation of and connectivity with word-related regions. Neuroimage, 2839-2849.

Galletti, C., Kutz, D. F., Gamberini, M., Breveglieri, R., \& Fattori, P. (2003). Role of the medial parieto-occipital cortex in the control of reaching and grasping movements. Experimental Brain Research, 153(2), 158-170.

Graves, W. W., Desai, R., Humphries, C., Seidenberg, M. S., \& Binder, J. R. (2010). Neural systems for reading aloud: A multiparametric approach. Cerebral Cortex, 20(8), 1799-1815.

Grill-Spector, K., Henson, R., \& Martin, A. (2006). Repetition and the brain: Neural models of stimulus-specific effects. Trends in Cognitive Sciences, 10(1), $14-23$.

Hagoort, P., Indefrey, P., Brown, C., Herzog, H., Steinmetz, H., \& Seitz, R. J. (1999). The neural circuitry involved in the reading of german words and pseudowords: A pet study. Jouranl of Cognitive Neuroscience, 11(4), 383-398.

Hashimoto, R., \& Sakai, K. L. (2004). Learning letters in adulthood: Direct visualization of cortical plasticity for forming a new link between orthography and phonology. Neuron, 42(2), 311-322.

Hastie, T., Tibshirani, R., \& Friedman, J. (2009). The elements of statistical learning: data mining, inference, and prediction ((2nd ed.). Springer.

Hayasaka, S., \& Nichols, T. E. (2003). Validating cluster size inference: Random field and permutation methods. Neuroimage, 20(4), 2343-2356.

Hickok, G., \& Poeppel, D. (2007). The cortical organization of speech processing. Nature Reviews Neuroscience, 8(5), 393-402.

Jobard, G., Crivello, F., \& Tzourio-Mazoyer, N. (2003). Evaluation of the dual route theory of reading: a metanalysis of 35 neuroimaging studies. Neuroimage, 20(2), $693-712$.

Joubert, S., Beauregard, M., Walter, N., Bourgouin, P., Beaudoin, G., Leroux, J.-M., et al. (2004). Neural correlates of lexical and sublexical processes in reading. Brain and Language, 89(1), 9-20.

Kravitz, D. J., Saleem, K. S., Baker, C. I., \& Mishkin, M. (2011). A new neural framework for visuospatial processing. Nature Reviews Neuroscience, 12(4), 217-230.

Levelt, W. J. (2001). Spoken word production: A theory of lexical access. Proceedings of the National Academy of Sciences of the United States of America, 98(23), $13464-13471$

Levelt, W. J., Roelofs, A., \& Meyer, A. S. (1999). A theory of lexical access in speech production. Behavioral and Brain Sciences, 22(1), 1-38 (discussion 38-75).

Levelt, W. J., \& Wheeldon, L. (1994). Do speakers have access to a mental syllabary? Cognition, 50(1-3), 239-269.

Mason, M. F., Norton, M. I., Van Horn, J. D., Wegner, D. M., Grafton, S. T., \& Macrae, C. N. (2007). Wandering minds: the default network and stimulus-independent thought. Science, 315(5810), 393-395.

McCandliss, B. D., Cohen, L., \& Dehaene, S. (2003). The visual word form area: expertise for reading in the fusiform gyrus. Trends in Cognitive Sciences, 7(7), 293-299.

Mochizuki-Kawai, H., Tsukiura, T., Mochizuki, S., \& Kawamura, M. (2006). Learningrelated changes of brain activation in the visual ventral stream: an fMRI study of mirror reading skill. Brain Research, 1122(1), 154-160.

Moser, D., Fridriksson, J., Bonilha, L., Healy, E. W., Baylis, G., Baker, J. M., et al. (2009). Neural recruitment for the production of native and novel speech sounds. Neuroimage, 46(2), 549-557.

Nazir, T. A., Jacobs, A. M., \& O'Regan, J. K. (1998). Letter legibility and visual word recognition. Memory and Cognition, 26(4), 810-821.

Penny, W. D., Holmes, A. P., \& Friston, K. J. (2003). Random effects analysis. In: R. S, J. Frackowiak, K. J. Friston, C. Frith, R. Dolan, C. J. Price, S. Zeki, J. Ashburner, \& W. D. Penny (Eds.), Human brain function (2nd ed.). Landon: Academic Press. 
Price, C. J. (2010). The anatomy of language: A review of $100 \mathrm{fMRI}$ studies published in 2009. Annals of the New York Academy of Science, 1191, 62-88.

Price, C. J., \& Devlin, J. T. (2011). The interactive account of ventral occipitotemporal contributions to reading. Trends in Cognitive Sciences, 15(6), 246-253.

Price, C. J., Green, D. W., \& von Studnitz, R. (1999). A functional imaging study of translation and language switching. Brain, 122(Pt 12), 2221-2235.

Pugh, K. R., Mencl, W. E., Jenner, A. R., Katz, L., Frost, S. J., Lee, J. R., et al. (2000). Functional neuroimaging studies of reading and reading disability (developmental dyslexia). Mental Retardation and Developmental Disabilities Research Reviews, 6(3), 207-213.

Raichle, M. E., \& Snyder, A. Z. (2007). A default mode of brain function: a brief history of an evolving idea. Neuroimage, 37(4), 1083-1090.

Sandak, R. Mencl, W. E., Frost, S. J., Rueckl, J. G., Katz, L., Moore, D. L., et al. (2004). The neurobiology of adaptive learning in reading: a contrast of different training conditions. Cognitive, Affective \& Behavioral Neuroscience, 4(1), 67-88.

Schacter, D. L., Addis, D. R., \& Buckner, R. L. (2007). Remembering the past to imagine the future: the prospective brain. Nature Reviews Neuroscience, $8(9)$, 657-661.

Schiller, N. O., Meyer, A. S., Baayen, R. H., \& Levelt, W. J. M. (1996). A comparison of lexeme and speech syllables in dutch. Journal of Quantitative Linguistics, 3, 8-28.

Seidenberg, M. S. (2007). Connectionist models of word reading. In: M. G. Gaskell (Ed.), Oxford handbook of psycholinguistics (pp. 235-250). Oxford: Oxford University Press.

Shuster, L. I. (2009). The effect of sublexical and lexical frequency on speech production: an fMRI investigation. Brain and Language, 111(1), 66-72.
Smith, E. E., Jonides, J., Marshuetz, C., \& Koeppe, R. A. (1998). Components of verbal working memory: Evidence from neuroimaging. Proceedings of the National Academy of Sciences of the United States of America, 95(3), 876-882.

Taylor, J. S. H., Rastle, K., \& Davis, M. H. (2012). Can cognitive models explain brain activation during word and pseudoword reading? A meta-analysis of 36 neuroimaging studies. Psychological Bulletin, 139(4), 766-791.

van Turennout, M., Bielamowicz, L., \& Martin, A. (2003). Modulation of neura activity during object naming: Effects of time and practice. Cerebral Cortex, 13 (4), 381-391.

Wilson, S. M., Isenberg, A. L., \& Hickok, G. (2009). Neural correlates of word production stages delineated by parametric modulation of psycholinguistic variables. Human Brain Mapping, 30(11), 3596-3608.

Xu, B., Grafman, J., Gaillard, W. D., Ishii, K., Vega-Bermudez, F., Pietrini, P., et al. (2001). Conjoint and extended neural networks for the computation of speech codes: the neural basis of selective impairment in reading words and pseudowords. Cerebral Cortex, 11(3), 267-277.

Xue, G., Chen, C., Jin, Z., \& Dong, Q. (2006a). Cerebral asymmetry in the fusiform areas predicted the efficiency of learning a new writing system. Journal of Cognitive Neuroscience, 18(6), 923-931.

Xue, G., Chen, C., Jin, Z. \& Dong, Q. (2006b). Language experience shapes fusiform activation when processing a logographic artificial language: an fMRI training study. Neuroimage, 31(3), 1315-1326.

Xue, G., \& Poldrack, R. A. (2007). The neural substrates of visual perceptual learning of words: implications for the visual word form area hypothesis. Journal of Cognitive Neuroscience, 19(10), 1643-1655 